\title{
Two-Party ECDSA from Hash Proof Systems and Efficient Instantiations
}

\author{
Guilhem Castagnos $^{1}$, Dario Catalano ${ }^{2}$, Fabien Laguillaumie $^{3}$, \\ Federico Savasta ${ }^{2,4}$, and Ida Tucker ${ }^{3}$ \\ 1 Université de Bordeaux, INRIA, CNRS, IMB UMR 5251, F-33405 Talence, France. \\ 2 Università di Catania, Italy. \\ 3 Univ Lyon, EnsL, UCBL, CNRS, Inria, LIP, F-69342, LYON Cedex 07, France. \\ 4 Scuola Superiore di Catania, Italy
}

\begin{abstract}
ECDSA is a widely adopted digital signature standard. Unfortunately, efficient distributed variants of this primitive are notoriously hard to achieve and known solutions often require expensive zero knowledge proofs to deal with malicious adversaries. For the two party case, Lindell [Lin17] recently managed to get an efficient solution which, to achieve simulation-based security, relies on an interactive, non standard, assumption on Paillier's cryptosystem. In this paper we generalize Lindell's solution using hash proof systems. The main advantage of our generic method is that it results in a simulation-based security proof without resorting to non-standard interactive assumptions.

Moving to concrete constructions, we show how to instantiate our framework using class groups of imaginary quadratic fields. Our implementations show that the practical impact of dropping such interactive assumptions is minimal. Indeed, while for 128-bit security our scheme is marginally slower than Lindell's, for 256-bit security it turns out to be better both in key generation and signing time. Moreover, in terms of communication cost, our implementation significantly reduces both the number of rounds and the transmitted bits without exception.
\end{abstract}

\section{Introduction}

Threshold cryptography Des88|DF90|GJKR96|SG98|Sho00 Boy86 $\mid$ CH89|MR04 allows $n$ users to share a common key in such a way that any subset of $t$ parties can use this key to decrypt or sign, while any coalition of less than $t$ can do nothing. The key feature of this paradigm is that it allows to use the shared key without explicitly reconstructing it in the clear. This means a subset of $t$ parties have to actively participate in the protocol whenever the secret key is used.

Applications of threshold cryptography range from contexts where many signers need to agree to sign one common document to distributed scenarios where sensitive documents should become accessible only by a quorum. This versatility sparked intense research efforts that, mainly in the decade from the early 1990s to the early 2000s, produced efficient threshold versions of most commonly used cryptographic schemes. Recent years have seen renewed interest in the field (e.g. [GGN16|Lin17|GG18|DKLs18|LN18|GG18|DKLs19]) for several reasons. First a number of start-up companies are using this technology to protect keys in real life applications Ser Unb Sep. Moreover, Bitcoin and other cryptocurrencies - for which security breaches can result in concrete financial losses - use ECDSA as underlying digital signature scheme. While multisignature-based countermeasures are built-in 
to Bitcoin, they offer less flexibility and introduce anonymity and scalability issues (see GGN16]). Finally, some of the schemes developed twenty years ago are not as efficient as current applications want them to be. This is the case, for instance, for ECDSA/DSA signatures. Indeed, while for many other schemes fast threshold variants are known (e.g. RSA decryption/signing and ECIES decryption) constructing efficient threshold variant of these signatures proved to be much harder. The main reason for this unfair distribution seems to result from the inversion step that requires one to compute $k^{-1} \bmod q$ from an unknown $k$. To explain why this is the case, let us first briefly recall how ECDSA actually works $5^{5}$. The public key is an elliptic curve point $Q$ and the signing key is $x$, such that $Q \leftarrow x P$, where $P$ is a generator of the elliptic curve group of points of order $q$. To sign a message $m$ one first hashes it using some suitable hash function $H$ and then proceeds according to the following algorithm

1. Choose $k$ random in $\mathbf{Z} / q \mathbf{Z}$

2. Compute $R \leftarrow k P$

3. Let $r \leftarrow r_{x} \bmod q$ where $R=\left(r_{x}, r_{y}\right)$

4. Set $s \leftarrow k^{-1}(H(m)+r x) \bmod q$

5. Output $(r, s)$

Now, the natural approach to make the above algorithm distributed would be to share $x$ additively among the participants and then start a multiparty computation protocol to produce the signature. In the two party case, this means that players start with shares $x_{1}$ and $x_{2}$ such that $Q=\left(x_{1}+x_{2}\right) P$. The players can then proceed by generating random shares $k_{1}, k_{2}$ such that $R=\left(k_{1}+k_{2}\right) P$. At this point, however, it is not clear how to compute, efficiently, shares $k_{1}^{\prime}, k_{2}^{\prime}$ such that $k_{1}^{\prime}+k_{2}^{\prime}=k^{\prime-1} \bmod q$.

Starting from [MR04 two party ECDSA signature protocols started adopting a less common multiplicative sharing both for $x$ and $k$. The basic idea of these constructions is very simple. Players start holding shares $x_{1}, x_{2}$ such that $Q=x_{1} x_{2} P=x P$. Whenever a new signature has to be generated they generate random $k_{1}, k_{2}$ such that $R=k_{1} k_{2} P=k P$. This immediately allows to get shares of the inverse $k^{\prime}$ as clearly $\left(k_{1}\right)^{-1}\left(k_{2}\right)^{-1}=\left(k_{1} k_{2}\right)^{-1} \bmod q$. As a final ingredient, the parties use Paillier's homomorphic encryption to secretly add their shares and complete the signature. For instance, player $P_{1}$ computes $c_{1} \leftarrow \operatorname{Enc}\left(\left(k_{1}\right)^{-1} H(m)\right)$ and $c_{2} \leftarrow \operatorname{Enc}\left(\left(k_{1}\right)^{-1} x_{1} r\right) . P_{2}$ can then complete the signature, using the homomorphic properties of the scheme as follows

$$
c \leftarrow c_{1}^{k_{2}^{-1}} c_{2}^{k_{2}^{-1} x_{2}}=\operatorname{Enc}\left(k^{-1} H(m)\right) \operatorname{Enc}\left(k^{-1} x r\right)=\operatorname{Enc}\left(k^{-1}(H(m)+x r)\right)
$$

$P_{2}$ concludes the protocol by sending back $c$ to $P_{1}$. Now, if $P_{1}$ also knows the decryption key, he can extract the signature $\left.s \leftarrow k^{-1}(H(m)+x r)\right)$ from $c$.

However, proving that each party followed the protocol correctly turns out to be hard. Initial attempts MR04 addressed this via expensive zero knowledge proofs. More recently Lindell in Lin17 managed to provide a much simpler and efficient protocol. The crucial idea of Lindell's protocol is the observation that, in the above two party ECDSA signing protocol, dishonest parties can create very little trouble. Indeed, if in a preliminary phase $P_{2}$ receives both Paillier's encryption key and an encryption $\operatorname{Enc}\left(x_{1}\right)$ of $P_{1}$ 's share of the secret signing key, essentially, all a corrupted $P_{1}$ can do is participate in the generation

\footnotetext{
${ }^{5}$ From now on we will focus on the elliptic curve variant of the scheme, as this is the most commonly used scheme in applications. We stress that our reasoning apply to the basic DSA case as well.
} 
of $R \leftarrow k_{1} k_{2} P$. Notice however that the latter is just the well established Diffie-Hellman protocol for which very efficient and robust protocols exist.

On the other hand, if $P_{2}$ is corrupted all she can do (except again participate in the generation of $R$ ) is to create a bad $c$ as a final response for $P_{1}$. However, while $P_{2}$ can certainly try that, this would be easy to detect by simply checking the validity of the resulting signature.

Turning this nice intuition into a formal proof induces some caveats though. A first problem comes from the fact that Paillier's plaintexts space is $\mathbf{Z} / N \mathbf{Z}$ ( $N$ is a large composite) whereas ECDSA signatures live in $\mathbf{Z} / q \mathbf{Z}$ ( $q$ is prime). Thus to avoid inconsistencies one needs to make sure that $N$ is taken large enough so that no wraps around occur during the whole signature generation process. This also means that, when sending $\operatorname{Enc}\left(x_{1}\right)$ to $P_{2}, P_{1}$ needs to prove that the plaintext $x_{1}$ is in the right range (i.e., sufficiently small).

A more subtle issue arises from the use of Paillier's encryption in the proof. Indeed, if one wants to use the scheme to argue indistinguishability of an adversary's view in real and simulated executions, it seems necessary to set up a reduction to the indistinguishability of Paillier's cryptosystem. This means one must design a proof technique that manages to successfully use Paillier's scheme without knowing the corresponding secret key. In Lindell's protocol the issue arises when designing the simulator's strategy against a corrupted player $P_{2}$. In such a case, $P_{2}$ might indeed send a wrong ciphertext $c$ (i.e., one that does not encrypt a signature) that the simulator simply cannot recognize as bad.

Lindell [Lin17] proposes two alternative proofs to overcome this. The first one relies on a game-based definition and avoids the problem by simply allowing the simulator to abort with a probability that depends on the number of issued signatures $q_{s}$. This results in a proof of security that is not tight (as the reduction actually looses a factor $q_{s}$ ). The second proof is simulation based, avoids the aborts, but requires the introduction of a new (interactive) non standard assumption regarding Paillier's encryption.

Thus, it is fair to say that, in spite of recent progress in the area, the following question remains open

Is it possible to devise a two party ECDSA signing protocol which is practical (both in terms of computational efficiency and in terms of bandwidth consumption), does not require interactive assumptions and allows for a tight security reduction.6

\subsection{Our contribution}

In this paper we provide a positive answer to the question above. In this sense, our contribution is twofold.

First, we provide a generic construction for two-party ECDSA signing from hash proof systems (HPS). Our solution can be seen as a generalization of Lindell's scheme [Lin17. to the general setting of HPSs that are homomorphic in the sense of [HO09]. This generic solution is not efficient enough for practical applications as, for instance, it employs general purpose zero knowledge as underlying building block. Still, beyond providing a clean, general framework which is of interest in its own right, it allows us to abstract away the properties we want to realize. In particular, our new protocol allows for a proof of security that is both tight and does not require artificial interactive assumptions when proving simulation

\footnotetext{
${ }^{6}$ We note here that the very recent two party protocol of DKLs18 is very fast in signing time and only relies on the ECDSA assumption. However its bandwidth consumption is much higher than Lin17.
} 
security. Indeed, in encryption schemes based on HPSs, indistinguishability of ciphertexts is not compromised by the challenger knowing the scheme's secret keys as it relies on a computational assumption and a statistical argument.

The correctness of our protocol follows from homomorphic properties that we require of the underlying HPS. We define the notion of homomorphically-extended projective hash families which ensure the homomorphic properties of the HPS hold for any public key sampled from an efficiently recognisable set, thus no zero-knowledge proofs are required for the public key.

Towards efficient solutions, we then show how to instantiate our (homomorphic) HPS construction using class groups of imaginary quadratic fields. Although the devastating attack from [CL09] shows that large families of protocols built over such groups are insecure, Castagnos and Laguillaumie CL15 showed that, if carefully designed, discrete logarithm based cryptosystems within such groups are still possible and allow for very efficient solutions. Algorithms to compute discrete logarithms in such groups have been extensively studied since the 80 's and the best ones known to date have a subexponential complexity 7 of $\mathcal{O}(L[1 / 2, o(1)])$ (compared to an $\mathcal{O}(L[1 / 3, o(1)])$ complexity for factorisation or discrete logarithm computation in finite fields). In [BH03], Bauer and Hamdy also showed that, for the specific case of imaginary quadratic fields, better complexities seem unlikely. Thus, the resulting schemes benefit from (asymptotically) shorter keys. Moreover, interest in the area has recently been renewed as it allows versatile and efficient solutions such as encryption switching protocols [CIL17, inner product functional encryption [CLT18] or verifiable delay functions [BBBF18 Wes19].

Concretely, the main feature of the Castagnos and Laguillaumie cryptosystem and its variants (CL from now on) is that they rely on the existence of groups with associated easy discrete log subgroups, for which hard decision problems can be defined. More precisely, in [CL15] there exist a cyclic group $G:=\langle g\rangle$ of order $q s$ where $s$ is unknown, $q$ is prime and $\operatorname{gcd}(q, s)=1$, and an associated cyclic subgroup of order $q, F:=\langle f\rangle$. Denoting with $G^{q}:=\left\langle g_{q}\right\rangle$ the subgroup of $q$-th powers in $G$ (of unknown order $s$ ), one has $G=F \times G_{p}$, and one can define an hard subgroup membership problem. Informally, and deferring for later the necessary mathematical details, this allows to build a linearly homomorphic encryption scheme where the plaintext space is $\mathbf{Z} / q \mathbf{Z}$ for arbitrarily large $q$. This also means that if one uses the very same $q$ underlying the ECDSA signature, one gets a concrete instantiation of our general protocol which naturally avoids all the inefficiencies resulting from $N$ and $q$ being different!

We remark that, similarly to Lindell's solution, our schemes require $P_{2}$ to hold an encryption $\operatorname{Enc}\left(x_{1}\right)$ of $P_{1}$ 's share of the secret key. As for Lindell's case, this imposes a somewhat heavy key registration phase in which $P_{1}$ has to prove, among other things, that the public key is correctly generated. While, in our setting we can achieve this without resorting to expensive range proofs, difficulties arise from the fact that (1) we work with groups of unknown order and (2) we cannot assume that all ciphertexts are valid (i.e., actually encrypt a message ${ }^{8}$. We address this by developing a new proof that solves both issues at the same time. Our proof is inspired by the Girault et al. [GPS06] identification protocol but introduces new ideas to adapt it to our setting and to make it a proof of knowledge. As for Lindell's case, it uses a binary challenge, which implies that the proof has to repeated $t$ times to get soundness error $2^{t}$. We believe that it should be possible to enlarge the chal-

${ }^{7} L[\alpha, c]$ denotes $L_{\alpha, c}(x):=\exp \left(c \log (x)^{\alpha} \log (\log (x))^{1-\alpha}\right)$

${ }^{8}$ For Paillier's scheme, used in Lin17, this is not an issue: every ciphertext is valid 
lenge space using techniques similar to those CKY09 adapted to work in the context of class groups. Exploring the actual feasibility of this idea is left as a future work. Clearly, advances in this direction would lead to substantial efficiency improvements.

As final contribution, we propose a $\mathrm{C}$ implementation of our protoco 9 . Our results show that our improved security guarantees come almost at no additional cost. Indeed, while our scheme is slightly slower (by a factor 1.5 for key generation and 3.5 for signing) for 128-bit security level, we are actually better for larger parameters: for 256-bit security, we are more efficient both in terms of key generation and signing time (by respective factors of 4.2 and 1.3). Intuitively, this behavior is due to the fact that our interactive key generation requires fewer exponentiations than that of Lindell's protocol (160 vs. 360), but an exponentiation in a class group is more expensive than an exponentiation in $\mathbf{Z} / n \mathbf{Z}$. The effect of the $L_{1 / 2}$ complexity and the fewer number of exponentiations starts at 192 bit of security. In terms of bandwidth, our protocol dramatically improves the communication cost by factors varying from 5 (112 bit security) to 10 (256 bit security) for key generation, and from 1.2 to 2.1 for signatures. It reduces the number of rounds from 175 (in Lindell's protocol) to 126 for the key generation process (the two signatures have the same number of rounds). We refer to Section 5 for precise implementation considerations and timings.

As a final remark, our HPS methods also allow a concrete implementation based on Paillier's decisional composite residuosity assumption, competitive with Lindell's for 112 and 128 bits of security as detailed in Appendix 6 .

\section{Preliminaries}

Notations. For a distribution $\mathscr{D}$, we write $d \hookleftarrow \mathscr{D}$ to refer to $d$ being sampled from $\mathscr{D}$ and

$b \stackrel{\$}{\leftarrow} B$ if $b$ is sampled uniformly in the set $B$. In an interactive protocol IP, between parties $P_{1}$ and $P_{2}$, we denote by $\mathrm{IP}\left\langle x_{1} ; x_{2}\right\rangle \rightarrow\left\langle y_{1} ; y_{2}\right\rangle$ the joint execution of parties $\left\{P_{i}\right\}_{i \in\{1,2\}}$ in the protocol, with respective inputs $x_{i}$, and where $P_{i}$ 's private output at the end of the execution is $y_{i}$.

The elliptic curve digital signature algorithm. ECDSA is the elliptic curve analogue of the Digital Signature Algoritm (DSA). It was put forth by Vanstone Van92 and accepted as ISO, ANSI, IEEE and FIPS standards. It works in a group $(\mathbb{G},+)$ of prime order $q$ (of say $\mu$ bits) of points of an elliptic curve over a finite field, generated by $P$ and consists of the following algorithms.

KeyGen $(\mathbb{G}, q, P) \rightarrow(x, Q)$ where $x \stackrel{\$}{\leftarrow} \mathbf{Z} / q \mathbf{Z}$ is the secret signing key and $Q \leftarrow x P$ is the public verification key.

$\operatorname{Sign}(x, m) \rightarrow(r, s)$ where $r$ and $s$ are computed as follows:

1. Compute $m^{\prime}$ : the $\mu$ leftmost bits of SHA256 $(m)$ where $m$ is to be signed.

2. Sample $k \stackrel{\$}{\leftarrow}(\mathbf{Z} / q \mathbf{Z})^{*}$ and compute $R \leftarrow k P$; denote $R=\left(r_{x}, r_{y}\right)$ and let $r \leftarrow r_{x}$ $\bmod q$. If $r=0$ chose another $k$.

3. Compute $s \leftarrow k^{-1} \cdot\left(m^{\prime}+r \cdot x\right) \bmod q$

$\operatorname{Verif}(Q, m,(r, s)) \rightarrow\{0,1\}$ indicating whether or not the signature is accepted.

\footnotetext{
${ }^{9}$ We also re-implemented Lindell's protocol to ensure a fair comparison
} 
Two-party ECDSA. This consists of the following interactive protocols:

IKeyGen $\langle(\mathbb{G}, q, P) ;(\mathbb{G}, q, P)\rangle \rightarrow\left\langle\left(x_{1}, Q\right) ;\left(x_{2}, Q\right)\right\rangle$ such that KeyGen $(\mathbb{G}, q, P) \rightarrow(x, Q)$ where $x_{1}$ and $x_{2}$ are shares of $x$.

$\operatorname{ISign}\left\langle\left(x_{1}, m\right) ;\left(x_{2}, m\right)\right\rangle \rightarrow\langle\emptyset ;(r, s)\rangle$ or $\langle(r, s) ; \emptyset\rangle$ or $\langle(r, s) ;(r, s)\rangle$ where $\emptyset$ is the empty output, signifying that one of the parties may have no output and $\operatorname{Sign}(x, m) \rightarrow(r, s)$.

The verification algorithm is non interactive and identical to that of ECDSA.

Interactive zero-knowledge proof systems. A zero-knowledge proof system $(P, V)$ for a language $\mathcal{L}$ is an interactive protocol between two probabilistic algorithms: a prover $P$ and a polynomial-time verifier $V$. Informally $\mathrm{P}$, detaining a witness for a given statement, must convince $V$ that it is true without revealing anything other to $V$. A more formal definition is provided in Appendix [

Simulation-based security and ideal functionalities. In order to prove a protocol is secure, one must first define what secure means. Basically, the Ideal/Real paradigm is to imagine what properties one would have in an ideal world; then if a real world (constructed) protocol has similar properties it is considered secure. We consider static adversaries, that choose which parties are corrupted before the protocol begins. For a detailed explanation of the simulation paradigm we refer the reader to [Lin16].

We will use ideal functionalities for commitments, zero-knowledge proofs of knowledge (ZKPoK) and commitments to non interactive zero-knowledge (NIZK) proofs of knowledge between two parties $P_{1}$ and $P_{2}$. We give the intuition behind these ideal functionalities with the example of ZKPoK. We consider the case of a prover $P_{i}$ with $i \in\{1,2\}$ who wants to prove the knowledge of a witness $w$ for an element $x$ which ensures that $(x, w)$ satisfy the relation R, i.e. $(x, w) \in \mathrm{R}$. In an ideal world we can imagine an honest and trustful third party, which can communicate with both $P_{i}$ and $P_{3-i}$. In this ideal scenario, $P_{i}$ could give $(x, w)$ to this trusted party, the latter would then check if $(x, w) \in \mathrm{R}$ and tell $P_{3-i}$ if this is true or false. In the real world we do not have such trusted parties and must substitute them with a cryptographic protocol between $P_{1}$ and $P_{2}$. Roughly speaking, the Ideal/Real paradigm requires that whatever information an adversary $\mathcal{A}$ (corrupting either $P_{1}$ or $P_{2}$ ) could recover in the real world, it can also recover in the ideal world. The trusted third party can be viewed as the ideal functionality and we denote it by $\mathcal{F}$. If some protocol satisfies the above property regarding this functionality, we call it secure.

Formally, we denote $\mathcal{F}\left\langle x_{1} ; x_{2}\right\rangle \rightarrow\left\langle y_{1} ; y_{2}\right\rangle$ the joint execution of the parties via the functionality $\mathcal{F}$, with respective inputs $x_{i}$, and respective private outputs at the end of the execution $y_{i}$. Each transmitted message is labelled with a session identifier sid, which identifies an iteration of the functionality. The ideal ZKPoK functionality [HL10, Section 6.5.3], denoted $\mathscr{F}_{\mathrm{zk}}$, is defined for a relation $\mathrm{R}$ by $\mathscr{F}_{\mathrm{zk}}\langle(x, w) ; \emptyset\rangle \rightarrow\langle\emptyset ;(x, \mathrm{R}(x, w))\rangle$, where $\emptyset$ is the empty output, signifying that the first party receives no output (cf. Fig. 1).

- Upon receiving (prove, sid, $x, w)$ from a party $P_{i}$ (for $i \in\{1,2\}$ ): if $(x, w) \notin \mathrm{R}$ or sid has been previously used then ignore the message. Otherwise, send (proof, sid, $x$ ) to party $P_{3-i}$

Fig. 1: The $\mathcal{F}_{\mathrm{zk}}^{\mathrm{R}}$ functionality 
The ideal commitment functionality, denoted $\mathscr{F}_{\text {com }}$, is depicted in Fig. 2 We also use an ideal functionality $\mathcal{F}_{\mathrm{com}-\mathrm{zk}}^{\mathrm{R}}$ for commitments to NIZK proofs for a relation R (cf. Fig. 3 . Essentially, this is a commitment functionality, where the committed value is a NIZK proof.

- Upon receiving (commit, sid, $x$ ) from party $P_{i}$ (for $i \in\{1,2\}$ ), record $($ sid, $i, x$ ) and send (receipt, sid) to party $P_{3-i}$. If some (commit, sid, $*$ ) is already stored, then ignore the message.

- Upon receiving (decommit, sid) from party $P_{i}$, if $(s i d, i, x)$ is recorded then send (decommit, sid, $x$ ) to party $P_{3-i}$.

Fig. 2: The $\mathscr{F}_{\text {com }}$ functionality

- Upon receiving (com - prove, sid, $x, w)$ from a party $P_{i}$ (for $i \in\{1,2\}$ ): if $(x, w) \notin \mathrm{R}$ or sid has been previously used then ignore the message. Otherwise, store $($ sid, $i, x)$ and send (proof - receipt, sid) to $P_{3-i}$.

- Upon receiving (decom - proof, sid) from a party $P_{i}$ (for $i \in\{1,2\}$ ): if $($ sid, $i, x)$ has been stored then send (decom - proof, sid, $x$ ) to $P_{3-i}$

Fig. 3: The $\mathscr{F}_{\mathrm{zk}-\mathrm{R} \text { (om }}^{\mathrm{R}}$ functionality

The ideal functionality for two-party ECDSA. The ideal functionality $\mathscr{F}_{E C D S A}$ (cf. Fig. 4) consists of two functions: a key generation function, called once, and a signing function, called an arbitrary number of times with the generated keys.

Consider an Elliptic-curve group $\mathbb{G}$ of order $q$ with generator a point $P$, then:

- Upon receiving $\operatorname{KeyGen}(\mathbb{G}, P, q)$ from both $P_{1}$ and $P_{2}$ :

1. Generate an $E C D S A$ key pair $(Q, x)$, where $x \stackrel{\$}{\leftarrow}(\mathbf{Z} / q \mathbf{Z})^{*}$ is chosen randomly and $Q$ is computed as $Q \leftarrow x \cdot P$.

2. Choose a hash function $H_{q}:\{0,1\}^{*} \rightarrow\{0,1\}^{\lfloor\log |q|\rfloor}$, and store $\left(\mathbb{G}, P, q, H_{q}, x\right)$.

3. Send $Q$ (and $H_{q}$ ) to both $P_{1}$ and $P_{2}$.

4. Ignore future calls to KeyGen.

- Upon receiving $\operatorname{Sign}\left(\right.$ sid, $m$ ) from both $P_{1}$ and $P_{2}$, where keys have already been generated from a call to Keygen and sid has not been previously used, compute an ECDSA signature $(r, s)$ on $m$, and send it to both $P_{1}$ and $P_{2}$. (To do this, choose a random $k \stackrel{\$}{\leftarrow}(\mathbf{Z} / q \mathbf{Z})^{*}$, compute $\left(r_{x}, r_{y}\right) \leftarrow k \cdot P$ and set $r \leftarrow r_{x} \bmod q$. Finally, compute $s \leftarrow k^{-1}\left(H_{q}(m)+r x\right)$ and output $(r, s)$.

Fig. 4: The $\mathscr{F}_{E C D S A}$ functionality 


\section{Two-Party ECDSA from Hash Proof Systems}

In this section we provide a generic construction for two-party ECDSA signing from hash proof systems (HPS) which we prove secure in the simulation based model. Throughout the section we consider the group of points of an elliptic curve $\mathbb{G}$ of order $q$, generated by $P$. In Subsection 3.1, we first recall some basic definitions on the HPS framework from CS02, before defining the specific properties we require for our construction in Subsection 3.2 in particular, to guarantee correctness of the protocol (in order for party $P_{2}$ to be able to perform homomorphic operations on ciphertexts provided by $P_{1}$, which are encryptions of elements in $\mathbf{Z} / q \mathbf{Z}$ ) the HPS must be homomorphic; and for security to hold against malicious adversaries we also require that the subset membership problem underlying the HPS be hard, and that the HPS be smooth. We note that diverse group systems (often used as a foundation for constructions of HPSs) imply all the aforementioned properties. Such HPSs define linearly homomorphic encryption schemes as described in Subsection 3.3 Finally, before presenting the overall two party signing protocol and proving its security, we describe the zero-knowledge proofs (ZKP) related to the aforementioned HPSs, and justify that they fulfil the $\mathscr{F}_{\text {com }} / \mathscr{F}_{\mathrm{zk} \text {-com }}$ hybrid model.

\subsection{Background on Hash Proof Systems}

Hash proof systems were introduced in [CS02] as a generalisation of the techniques used in CS98 to design chosen ciphertext secure public-key encryption schemes. Consider a set of words $\mathcal{X}$, an NP language $\mathcal{L} \subset \mathcal{X}$ such that $\mathcal{L}:=\{x \in \mathcal{X} \mid w \in \mathscr{W}:(x, w) \in \mathrm{R}\}$ where $\mathrm{R}$ is the relation defining the language, $\mathcal{L}$ is the language of true statements in $\mathcal{X}$, and for $(x, w) \in \mathrm{R}, w \in \mathscr{W}$ is a witness for $x \in \mathcal{L}$. The set $(\mathcal{X}, \mathcal{L}, \mathfrak{W}, \mathrm{R})$ defines an instance of a subset membership problem, i.e. the problem of deciding if an element $x \in \mathscr{X}$ is in $\mathscr{L}$ or in $x \backslash \mathcal{L}$.

A HPS associates a projective hash family (PHF) to such a subset membership problem. The PHF defines a key generation algorithm PHF.KeyGen which outputs a secret hashing key hk sampled from distribution of hashing keys $\mathscr{D}_{\text {hk }}$ over a hash key space $K_{\text {hk }}$, and a public projection key hp $\leftarrow \alpha(\mathrm{hk})$ in projection key space $K_{\mathrm{hp}}$ (where $\alpha: K_{\mathrm{hk}} \mapsto K_{\mathrm{hp}}$ is an efficient auxiliary function). The secret hashing key hk defines a hash function $\mathcal{H}_{\mathrm{hk}}$ : $X \mapsto \Pi$, and hp allows for the (public) evaluation of the hash function on words $x \in \mathcal{L}$, i.e. $\mathscr{H}_{\mathrm{hp}}(x, w)=\mathscr{H}_{\mathrm{hk}}(x)$ for $(x, w) \in \mathrm{R}$. A projective hash family PHF is thus defined by PHF $:=\left(\left\{\mathcal{H}_{\mathrm{hk}}\right\}_{\mathrm{hk} \in K_{\mathrm{hk}}}, K_{\mathrm{hk}}, \mathcal{X}, \mathcal{L}, \Pi, K_{\mathrm{hp}}, \alpha\right)$.

\subsection{Required Properties}

$\delta_{s}$-smoothness. The standard smoothness property of a PHF requires that for any $x \notin \mathcal{L}$, the value $\mathcal{H}_{\mathrm{hk}}(x)$ be uniformly distributed knowing hp. In this work messages will be encoded in a subgroup $\mathcal{F}$ of $\Pi$ of order $q$, indeed, for integration with ECDSA it must hold that the group in which the message is encoded has order $q$, since the message space is dictated by the order of the elliptic curve group $\mathbb{G}$. In some instantiations $\mathscr{F}=\Pi$, but $\mathscr{F}$ may also be a strict subgroup of $\Pi$. For $m \in \mathbf{Z} / q \mathbf{Z}$ we denote $\operatorname{Encode}(m)$ the encoding of $m$ in $\mathcal{F}$, where Encode : $(\mathbf{Z} / q \mathbf{Z},+) \mapsto(\mathcal{F}, \cdot)$ is an efficient isomorphism. We denote Decode the inverse isomorphism, which must also be efficiently computable.

If $\mathscr{F} \subsetneq \Pi$, we require smoothness over $\mathcal{X}$ on $\mathscr{F}$ [CS02, Subsection 8.2.4]. A projective hash family is $\delta_{s}$-smooth over $\mathcal{X}$ on $\mathscr{F}$ if for any $x \in \mathcal{X} \backslash \mathcal{L}$, a random $\pi \in \mathcal{F}$ and a 
randomly sampled hashing key hk $\hookleftarrow \mathscr{D}_{\mathrm{hk}}$, the distributions $U:=\left\{x, \alpha(\mathrm{hk}), \pi \cdot \mathscr{H}_{\mathrm{hk}}(x)\right\}$ and $V:=\left\{x, \alpha(\mathrm{hk}), \mathcal{H}_{\mathrm{hk}}(x)\right\}$ are $\delta_{s}$-close.

$\delta_{\mathcal{L}}-$ hard subset membership problem. For security to hold $(\mathcal{X}, \mathcal{L}, \mathfrak{W}, \mathrm{R})$ must be an instance of a hard subset membership problem, i.e. no polynomial time algorithm can distinguish

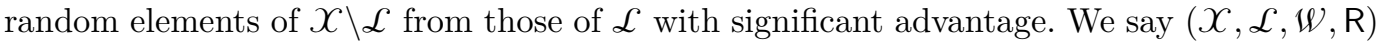
is a $\delta_{\mathscr{L}}$-hard subset membership problem if $\delta_{\mathscr{L}}$ is the maximal advantage of any polynomial time adversary in distinguishing random elements of $\mathcal{X} \backslash \mathcal{L}$ from those of $\mathcal{L}$.

Linearly homomorphic PHF. In order for the homomorphic operations performed by $P_{2}$ to hold in the two party ECDSA protocol, we require that the projective hash family also be homomorphic as defined in [HO09].

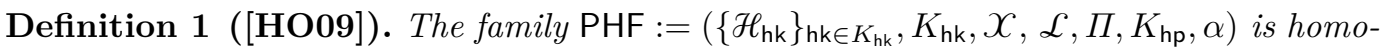
morphic if $(X, \star)$ and $(\Pi, \cdot)$ are groups, and for all $\mathrm{hk} \in K_{\mathrm{hk}}$, and $u_{1}, u_{2} \in \mathcal{X}$, we have $\mathcal{H}_{\mathrm{hk}}\left(u_{1}\right) \cdot \mathcal{H}_{\mathrm{hk}}\left(u_{2}\right)=\mathcal{H}_{\mathrm{hk}}\left(u_{1} \star u_{2}\right)$, that is to say $\mathcal{H}_{\mathrm{hk}}$ is a homomorphism for each hk.

This clearly implies that for $\mathrm{hp} \leftarrow \alpha$ (hk) the public projective hash function is linearly homomorphic with respect to elements $u_{1}, u_{2} \in \mathcal{L}$.

Homomorphically extended PHF. Note that the co-domain of $\alpha$, which specifies the set of valid projection keys, may not be efficiently recognisable. Though we do not require - as did the protocol of [Lin17] - a costly ZKPoK of the secret key associated to the public key, it is essential in our protocol that even if a public key is chosen maliciously (i.e. there does not exist hk $\in K_{\text {hk }}$ such that hp $\leftarrow \alpha(\mathrm{hk})$, which may go unnoticed to honest parties in the protocol), the homomorphic properties of the public projective hash function still hold. We thus require that the co-domain of $\alpha$, which defines valid projection keys, be contained in an efficiently recognisable space $K_{\mathrm{hp}}^{\prime}$, such that for all $\mathrm{hp}^{\prime} \in K_{\mathrm{hp}}^{\prime}, \mathcal{H}_{\mathrm{hp}^{\prime}}$ is a homomorphism (respectively to its inputs in $\mathcal{L}$ ).

Definition 2 (Homomorphically extended PHF). We say that the family PHF := $\left(\left\{\mathcal{H}_{\mathrm{hk}}\right\}_{\mathrm{hk} \in K_{\mathrm{hk}}}, K_{\mathrm{hk}}, \mathcal{X}, \mathcal{L}, \Pi, K_{\mathrm{hp}}, K_{\mathrm{hp}}^{\prime}, \alpha\right)$ is homomorphically extended if $\mathrm{PHF}:=\left(\left\{\mathcal{H}_{\mathrm{hk}}\right\}_{\mathrm{hk} \in K_{\mathrm{hk}}}\right.$, $\left.K_{\mathrm{hk}}, \mathcal{X}, \mathcal{L}, \Pi, K_{\mathrm{hp}}, \alpha\right)$ is a homomorphic PHF and that there exists an efficiently recognizable space $K_{\mathrm{hp}}^{\prime} \supseteq K_{\mathrm{hp}}$ such that for any $\mathrm{hp}^{\prime} \in K_{\mathrm{hp}}^{\prime}$, the projective hash function associated to $\mathrm{hp}$ is a homomorphism (respectively to its inputs in $\mathcal{L}$ ).

ECDSA-friendly HPS. We here define the notion of an ECDSA-friendly HPS, essentially it is a HPS which meets all of the aforementioned properties, and which suffices to ensure simulation based security in the protocol of Subsection 3.5

Definition $3\left(\delta_{s} / \delta_{\mathcal{L}}\right.$-ECDSA-friendly HPS). Let $\mathcal{X}, \Pi$ and $\mathcal{F}$ be groups such that $\mathcal{F}$ is a subgroup of $\Pi$ of prime order $q$, and such that there exists an efficient isomorphism Encode $:(\mathbf{Z} / q \mathbf{Z},+) \mapsto(\mathcal{F}, \cdot)$, whose inverse Decode is also efficiently computable. A $\delta_{s} / \delta_{\mathcal{L}}-E C D S A$-friendly hash proof system is a hash proof system which associates to a $\delta_{\mathcal{L}}$-hard subset membership problem a homomorphically extended projective hash family PHF $:=\left(\left\{\mathcal{H}_{\mathrm{hk}}\right\}_{\mathrm{hk} \in K_{\mathrm{hk}}}, K_{\mathrm{hk}}, \mathcal{X}, \mathcal{L}, \Pi, K_{\mathrm{hp}}, K_{\mathrm{hp}}^{\prime}, \alpha\right)$ which is $\delta_{s}-$ smooth over $\mathcal{X}$ on $\mathcal{F}$. 


\subsection{Resulting Encryption Scheme}

We use the standard chosen plaintext attack secure encryption scheme which results from a HPS [CS02. The key generation algorithm simply runs PHF.KeyGen and sets $h k \in K_{\mathrm{hk}}$ as the secret key, and the associated public key is $\mathrm{hp} \leftarrow \alpha(\mathrm{hk})$. Encryption of a plaintext message $m$ in $\mathbf{Z} / q \mathbf{Z}$ is done by sampling a random pair $(u, w) \in \mathbf{R}$ and computing $\mathrm{Enc}(\mathrm{hp}, m) \leftarrow$ $\left(u, \mathcal{H}_{\mathrm{hp}}(u, w) \cdot \operatorname{Encode}(m)\right)$. To specify the randomness used in the encryption algorithm, we sometimes use the notation $\operatorname{Enc}((u, w) ;(\mathrm{hp}, m))$. To decrypt a ciphertext $(u, e) \in X \times \Pi$ with secret key hk do: $\operatorname{Dec}(\mathrm{hk},(u, e)) \leftarrow \operatorname{Decode}\left(\frac{e}{\mathscr{H}_{\mathrm{hk}}(u)}\right)$. The scheme is indistinguishable under chosen plaintext attacks assuming both the smoothness of the HPS and the hardness of the underlying subset membership problem.

Homomorphic properties. Given encryptions $\left(u_{1}, e_{1}\right)$ and $\left(u_{2}, e_{2}\right)$ of respectively $m_{1}$ and $m_{2}$, and an integer $a$, we require that there exist two procedures EvalSum and EvalScal such that $\operatorname{Dec}\left(\mathrm{hk}, \operatorname{EvalSum}\left(\mathrm{hp},\left(u_{1}, e_{1}\right),\left(u_{2}, e_{2}\right)\right)\right)=m_{1}+m_{2}$ and $\operatorname{Dec}\left(\mathrm{hk}\right.$, EvalScal $\left.\left(\mathrm{hp},\left(u_{1}, e_{1}\right), a\right)\right)=$ $a \cdot m_{1}$; which is the case if the projective hash family is homomorphic.

\subsection{Zero-Knowledge Proofs}

Proofs of knowledge. We use the $\mathscr{F}_{\mathrm{zk}}, \mathscr{F}_{\mathrm{zk}-\mathrm{com}}$ hybrid model. Ideal ZK functionalities are used for the following relations, were the parameters of the elliptic curve $(\mathbb{G}, P, q)$ are implicit public inputs:

1. $R_{D L}:=\{(Q, w) \mid Q=w P\}$, proves the knowledge of the discrete log of an elliptic curve point.

2. $R_{\mathrm{HPS}-\mathrm{DL}}:=\left\{\left(\mathrm{hp},\left(c_{1}, c_{2}\right), Q_{1}\right) ;\left(x_{1}, w\right) \mid\left(c_{1}, c_{2}\right)=\operatorname{Enc}\left((u, w) ;\left(\mathrm{hp}, x_{1}\right)\right) \wedge\left(c_{1}, w\right) \in \mathrm{R} \wedge Q_{1}=\right.$ $\left.x_{1} P\right\}$, proves the knowledge of the randomness used for encryption, and of the value $x_{1}$ which is both encrypted in the ciphertext $\left(c_{1}, c_{2}\right)$ and the discrete log of the elliptic curve point $Q_{1}$.

The functionalities $\mathcal{F}_{\mathrm{zk}}^{R_{D L}}, \mathcal{F}_{\mathrm{zk}-\mathrm{com}}^{R_{D L}}$ can be instantiated using Schnorr proofs Sch91. For

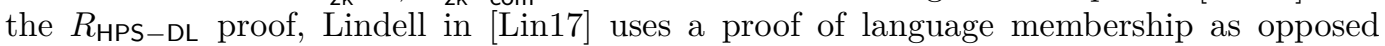
to a proof of knowledge. Though his technique is quite generic, it cannot be used in our setting. Indeed, his approach requires that the ciphertext be valid, which means that the element $c$ must be decryptable. As Lindell uses Paillier's encryption scheme, any element of $\left(\mathbf{Z} / N^{2} \mathbf{Z}\right)^{\times}$is a valid ciphertext. This is not the case for a HPS-based encryption scheme, since it incorporates redundancy so that any pair in $X \times \Pi$ is not a valid ciphertext.

For our instantiations, we will introduce specific and efficient proofs. Note that in any case, we needn't prove that $x_{1} \in \mathbf{Z} / q \mathbf{Z}$ since both the message space of our encryption scheme and the elliptic curve group $\mathbb{G}$ are of order $q$.

\subsection{Two-Party ECDSA Signing Protocol with Simulation-Based Security}

We here provide our generic construction for two-party ECDSA signing from hash proof systems (Figure 5), along with a proof that the protocol is secure in the Ideal/Real paradigm (Theorem 1). To this end, we must argue the indistinguishability of an adversary's view corrupting either party $P_{1}$ or $P_{2}$ - in real and simulated executions. In Cramer-Shoup like encryption schemes (resulting from HPSs as described in Subsection 3.3), the chosen plaintext attack indistinguishability of ciphertexts allows for the challenger in the security 
game to sample the secret hashing key hk, and compute the resulting projection key hp. Thus hk is known to the challenger. Indeed here, in order to prove indistinguishability, the challenger first replaces the random masking element $u \in \mathcal{L}$ in the original encryption scheme with an element sampled outside the language $u^{\prime} \in \mathcal{C} \backslash \mathcal{L}$. Note that in order to perform this change the challenger must know the secret hashing key. The hardness of the subset membership problem ensures this goes unnoticed to any polynomial time adversary. Then the smoothness of the projective hash family allows one to replace the plaintext value by some random element from the plaintext space, thus guaranteeing the indistinguishability of the resulting encryption scheme. We insist on this point since in Lindell's protocol [Lin17, many issues arise from the use of Paillier's cryptosystem, for which the indistinguishability of ciphertexts no longer holds if the challenger knows the secret key. In particular this implies that in Lindell's game based proof, instead of letting the simulator use the Paillier secret key to decrypt the incoming ciphertext (and check the corrupted party $P_{2}$ did not send a different ciphertext $c$ than that prescribed by the protocol), the simulator guesses when the adversary may have cheated by simulating an abort with a probability depending on the number of issued signatures. This results in a proof of security which is not tight.

Moreover, though this technique suffices for a game-based definition, it does not for simulation-based security definitions. Thus, in order to be able to prove their protocol using simulation, they use a rather non-standard assumption, called Paillier-EC assumption and recalled in Appendix IV] Thanks to the framework we have chosen to adopt, we are able to avoid such an artificial interactive assumption. Moreover, should one write a game based proof for our construction, the security loss present in [Lin17] would not appear.

Finally we note that the correctness of our protocol follows from the fact Encode is an efficient isomorphism, and from the fact the hash function is linearly homomorphic for any public key in the efficiently recognisable space $K_{\mathrm{hp}}^{\prime}$.

Theorem 1. Assume HPS is a $\delta_{s} / \delta_{\alpha}-E C D S A$-friendly HPS then the protocol of Figure 5 securely computes $\mathscr{F}_{E C D S A}$ in the $\left(\mathscr{F}_{\mathrm{zk}}, \mathscr{F}_{\mathrm{com}-\mathrm{zk}}\right)$-hybrid model in the presence of a malicious static adversary (under the ideal/real definition). Indeed there exists a simulator for the scheme such that no polynomial time adversary - having corrupted either $P_{1}$ or $P_{2}-$ can distinguish a real execution of the protocol from a simulated one with probability greater than $2 \delta_{\alpha}+\delta_{s}$.

Proof. In this proof, the simulator $\mathcal{S}$ only has access to an ideal functionality $\mathscr{F}_{E C D S A}$ for computing ECDSA signatures, so all it learns in the ideal world is the public key $Q$ which it gets as output of the KeyGen phase from $\mathscr{F}_{E C D S A}$ and signatures $(r, s)$ for messages $m$ of its choice as output of the Sign phase. However in the real world, the adversary, having either corrupted $P_{1}$ or $P_{2}$ will also see all the interactions with the non corrupted party which lead to the computation of a signature. Thus $\mathcal{S}$ must be ale to simulate $\mathcal{A}$ 's view of these interactions, while only knowing the expected output. To this end $\delta$ must set up with $\mathcal{A}$ the same public key $Q$ that it received from $\mathscr{F}_{E C D S A}$, in order to be able to subsequently simulate interactively signing messages with $\mathcal{A}$, using the output of $\mathscr{F}_{E C D S A}$ from the Sign phase.

$\mathcal{S}$ simulates $P_{2}-$ Corrupted $P_{1}$ : We first show that if an adversary $\mathcal{A}_{1}$ corrupts $P_{1}$, one can construct a simulator $\mathcal{S}$ s.t. the output distribution of $\mathcal{S}$ is indistinguishable from $\mathcal{A}_{1}$ 's view in an interaction with an honest party $P_{2}$. The main difference here with the proof of Lin17] arises from the fact we no longer use a ZKP from which $\delta$ can extract the encryption scheme's secret key. Instead, $\delta$ extracts the randomness used for encryption and the plaintext 


\begin{tabular}{|c|c|c|}
\hline$P_{1}$ & IKeyGen $(\mathbb{G}, P, q)$ & $P_{2}$ \\
\hline \multicolumn{3}{|l|}{$x_{1} \stackrel{\$}{\leftarrow} \mathbf{Z} / q \mathbf{Z}$} \\
\hline \multirow[t]{2}{*}{$Q_{1} \leftarrow x_{1} P$} & $\stackrel{\left(\text { com-prove }, 1, Q_{1}, x_{1}\right)}{\longrightarrow} \mathcal{F}_{\mathrm{zk}-\mathrm{com}}^{R_{D L}} \stackrel{\text { (proof-receipt }, 1)}{\longrightarrow}$ & \\
\hline & & $x_{2} \stackrel{\$}{\leftarrow} \mathbf{Z} / q \mathbf{Z}$ \\
\hline \multirow[t]{2}{*}{$\begin{array}{l}P_{1} \text { aborts if (proof, } 2, Q_{2} \text { ) not re- } \\
\text { ceived. }\end{array}$} & $\stackrel{\left(\text { proof }, 2, Q_{2}\right)}{\longleftarrow} \mathscr{F}_{\mathrm{zk}}^{R_{D L}} \stackrel{\left(\text { prove }, 2, Q_{2}, x_{2}\right)}{\longleftarrow}$ & $Q_{2} \leftarrow x_{2} P$ \\
\hline & $\stackrel{\text { (decom-proof,1) }}{\longrightarrow} \mathcal{F}_{z-c}^{R_{D L}} \stackrel{\left.\text { (decom-proof, } 1, Q_{1}\right)}{\longrightarrow}$ & \\
\hline \multicolumn{3}{|l|}{$\mathrm{hk} \hookleftarrow \mathscr{D}_{\mathrm{hk}} ; \mathrm{hp} \leftarrow \alpha(\mathrm{hk})$} \\
\hline \multicolumn{3}{|l|}{ Sample $(u, w) \in \mathbf{R}$} \\
\hline \multicolumn{3}{|c|}{ 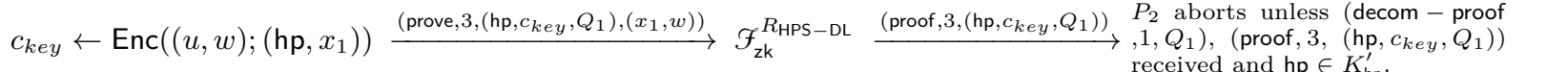 } \\
\hline \multicolumn{2}{|l|}{$Q \leftarrow x_{1} Q_{2}$} & $Q \leftarrow x_{2} Q_{1}$ \\
\hline$P_{1}$ & $\operatorname{ISign}(m, s i d)$ & $P_{2}$ \\
\hline \multicolumn{3}{|l|}{$k_{1} \stackrel{\$}{\leftarrow} \mathbf{Z} / q \mathbf{Z}$} \\
\hline$R_{1} \leftarrow k_{1} P$ & $\stackrel{\left(\text { com-prove }, \text { sid } \| 1, R_{1}, k_{1}\right)}{\longrightarrow} \mathcal{F}_{\mathrm{zk}-\mathrm{com}}^{R_{D L}} \stackrel{\text { (proof-receipt, sid } \| 1)}{\longrightarrow}$ & $\begin{array}{l}k_{2} \leftarrow \mathbf{Z} / q \mathbf{Z} \\
R_{2} \leftarrow k_{2} P\end{array}$ \\
\hline $\begin{array}{l}P_{1} \text { aborts if (proof, sid }|| 2, R_{2} \text { ) } \\
\text { not received. }\end{array}$ & 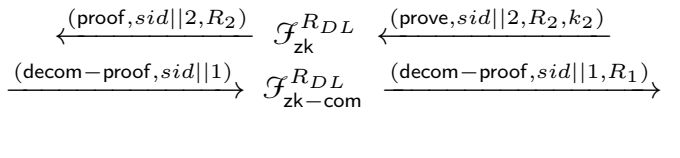 & $\begin{array}{l}P_{2} \text { aborts if }(\text { decom }- \text { proof } \\
\left.\text { sid } \| 1, R_{1}\right) \text { not received. } \\
m^{\prime} \leftarrow H(m)\end{array}$ \\
\hline $\begin{array}{l}R=\left(r_{x}, r_{y}\right) \leftarrow k_{1} R_{2} \\
\quad r \leftarrow r_{x} \bmod q\end{array}$ & & $\begin{array}{c}R=\left(r_{x}, r_{y}\right) \leftarrow k_{2} R_{1} \\
r \leftarrow r_{x} \bmod q \\
c_{1} \leftarrow \operatorname{Enc}\left(\mathrm{hp}, k_{2}^{-1} \cdot m^{\prime}\right) \\
c_{2} \leftarrow \operatorname{EvalScal}\left(\mathrm{hp}, c_{k e y}, k_{2}^{-1} \cdot r \cdot x_{2}\right)\end{array}$ \\
\hline $\begin{array}{c}\alpha \leftarrow \operatorname{Dec}\left(\mathrm{hk}, c_{3}\right) \\
\hat{s} \leftarrow \alpha \cdot k_{1}^{-1}, s \leftarrow \min (\hat{s}, q-\hat{s}) \\
\text { If not Verif }(Q, m,(r, s)) P_{1} \text { aborts } \\
\text { else Return }(r, s)\end{array}$ & $\longleftarrow c_{3}$ & $c_{3} \leftarrow \operatorname{EvalSum}\left(\mathrm{hp}, c_{1}, c_{2}\right)$ \\
\hline
\end{tabular}

Fig. 5: Two-Party ECDSA Key Generation and Signing Protocols from HPSs 
$x_{1}$ from the ZKPoK for $R_{\mathrm{HPS}-\mathrm{DL}}$, which allows it to recompute the ciphertext and verify it obtains the expected value $c_{k e y}$. Moreover since the message space of our encryption scheme is $\mathbf{Z} / q \mathbf{Z}$, if $\mathcal{A}_{1}$ does not cheat in the proofs (which is guaranteed by the $\left(\mathcal{F}_{z \mathrm{k}}, \mathscr{F}_{\text {com-zk }}\right.$ )-hybrid model), the obtained distributions are identical in the ideal and real executions (as opposed to statistically close as in [Lin17]).

\section{Key Generation Phase}

1. Given input $\operatorname{KeyGen}(\mathbb{G}, P, q)$, the simulator $\delta$ sends $\operatorname{KeyGen}(\mathbb{G}, P, q)$ to the ideal functionality $\mathscr{F}_{E C D S A}$ and receives back a public key $Q$.

2. $\mathcal{S}$ invokes $\mathcal{A}_{1}$ on input IKeyGen $(\mathbb{G}, P, q)$ and receives the commitment to a proof of knowledge of $x_{1}$ such that $Q_{1}=x_{1} P$ denoted (com - prove, $\left.1, Q_{1}, x_{1}\right)$ as $\mathcal{A}_{1}$ intends to send to $\mathscr{F}_{\mathrm{zk}-\mathrm{com}}^{R_{D L}}$, such that $\mathcal{S}$ can extract $x_{1}$ and $Q_{1}$.

3. Using the extracted value $x_{1}, \mathcal{S}$ verifies that $Q_{1}=x_{1} P$. If so, it computes $Q_{2} \leftarrow x_{1}^{-1} Q$ (using the value $Q$ received from $\mathscr{F}_{E C D S A}$ ); otherwise $\mathcal{S}$ samples a random $Q_{2}$ from $\mathbb{G}$.

4. $\mathcal{S}$ sends (proof, $2, Q_{2}$ ) to $\mathcal{A}_{1}$ as if sent by $\mathcal{F}_{\mathrm{zk}}^{R_{D L}}$ thereby $\delta$ simulating a proof of knowledge of $x_{2}$ s.t. $Q_{2}=x_{2} P$.

5. $\delta$ receives (decom - proof, 1 ) as $\mathcal{A}_{1}$ intends to send to $\mathcal{F}_{\mathrm{zk}-\mathrm{com}}^{R_{D L}}$ and simulates $P_{2}$ aborting if $Q_{1} \neq x_{1} P$. $\mathcal{S}$ also receives (prove, $3,\left(\mathrm{hp}, c_{k e y}, Q_{1}\right),\left(x_{1}, w\right)$ ) as $\mathcal{A}_{1}$ intends to send to $\mathcal{F}_{\mathrm{zk}}^{R_{\mathrm{HPS}-\mathrm{DL}}}$.

6. $\delta$ computes $u$ from $w$ such that $(u, w) \in \mathrm{R}$, and using the extracted value $x_{1}$ verifies that $c_{k e y}=\operatorname{Enc}\left((u, w) ;\left(\mathrm{hp}, x_{1}\right)\right)$, and simulates $P_{2}$ aborting if not.

7. $\mathcal{S}$ sends continue to $\mathcal{F}_{E C D S A}$ for $P_{2}$ to receive output, and stores $\left(x_{1}, Q, c_{k e y}\right)$.

When taking $\mathscr{F}_{\mathrm{zk}}$ and $\mathscr{F}_{\mathrm{com}-\mathrm{zk}}$ as ideal functionalities, the only difference between the real execution as ran by an honest $P_{2}$, and the ideal execution simulated by $\delta$ is that in the former $Q_{2} \leftarrow x_{2} P$ where $x_{2} \stackrel{\$}{\leftarrow} / q \mathbf{Z}$, whereas in the latter $Q_{2} \leftarrow x_{1}^{-1} Q$, where $Q$ is the public key returned by the ideal functionality $\mathscr{F}_{E C D S A}$. However since $\mathfrak{F}_{E C D S A}$ samples $Q$ uniformly at random from $\mathbb{G}$, the distribution of $Q_{2}$ in both cases is identical.

\section{Signing Phase}

1. Upon input $\operatorname{Sign}(\operatorname{sid}, m)$, simulator $\mathcal{S}$ sends $\operatorname{Sign}(\operatorname{sid}, m)$ to $\mathscr{F}_{E C D S A}$ and receives back a signature $(r, s)$.

2. $\delta$ computes the elliptic curve point $R=\left(r, r_{y}\right)$ using the ECDSA verification algorithm.

3. $\mathcal{S}$ invokes $\mathcal{A}_{1}$ with input ISign $($ sid, $m)$ and simulates the first three interactions such that $\mathcal{A}_{1}$ computes $R$. The strategy is similar to that used to compute $Q$, in brief, it proceeds as follows:

(a) $\mathcal{S}$ receives (com - prove, sid || $1, R_{1}, k_{1}$ ) from $\mathcal{A}_{1}$.

(b) If $R_{1}=k_{1} P$ then $\delta$ sets $R_{2} \leftarrow k_{1}^{-1} R$; otherwise it chooses $R_{2}$ at random. $\delta$ sends (proof, sid $\left.\| 2, R_{2}\right)$ to $\mathcal{A}_{1}$.

(c) $\mathcal{S}$ receives (decom - proof, sid $\| 1$ ) from $\mathcal{A}_{1}$. If $R_{1} \neq k_{1} P$ then $\delta$ simulates $P_{2}$ aborting and instructs the trusted party computing $\mathscr{F}_{E C D S A}$ to abort.

4. $\delta$ computes $c_{3} \leftarrow \operatorname{Enc}_{p k}\left(k_{1} \cdot s \bmod q\right)$, where $s$ was received from $\mathscr{F}_{E C D S A}$, and sends $c_{3}$ to $\mathcal{A}_{1}$.

As with the computation of $Q_{2}$ in the key generation phase, $R_{2}$ is distributed identically in the real and ideal executions since $R$ is randomly generated by $\mathscr{F}_{E C D S A}$. The zeroknowledge proofs and verifications are also identically distributed in the $\mathscr{F}_{\mathrm{zk}}, \mathscr{F}_{\mathrm{com}-\mathrm{zk}}$-hybrid model. Thus, the only difference between a real execution and the simulation is the way 
that $c_{3}$ is computed. In the simulation it is an encryption of $k_{1} \cdot s=k_{1} \cdot k^{-1}\left(m^{\prime}+r \cdot x\right)=$ $k_{2}^{-1} \cdot\left(m^{\prime}+r \cdot x\right) \bmod q$, whereas in a real execution $c_{3}$ is computed from $c_{k e y}$, using the homomorphic properties of the encryption scheme. However, notice that as long as there exist $(u, w)$ such that $c_{k e y}=\operatorname{Enc}\left((u, w) ;\left(\mathrm{hp}, x_{1}\right)\right)$ where $Q=x_{1} P$ - which is guaranteed by the ideal functionality $\mathcal{F}_{\mathrm{zk}}^{R_{\mathrm{HPS}} \mathrm{DL}}$ - and as long as the homomorphic operations hold - which is guaranteed for any hp in the efficiently verifiable ensemble $K_{\mathrm{hp}}^{\prime}$ (cf. Subsection 3.2 - the $c_{3}$ obtained in the real scenario is also an encryption of $s^{\prime}=k_{2}^{-1} \cdot\left(m^{\prime}+r \cdot x\right) \bmod q$. Thus $c_{3}$ is distributed identically in both cases.

This implies that the view of a corrupted $P_{1}$ is identical in the real and ideal executions of the protocol (in the $\mathscr{F}_{\mathrm{zk}}, \mathscr{F}_{\mathrm{com}-\mathrm{zk}}$-hybrid model), i.e., the simulator perfectly simulates the real environment, which completes the proof of this simulation case.

$\delta$ simulates $P_{1}-$ Corrupted $P_{2}$ : We now suppose an adversary $\mathcal{A}_{2}$ corrupts $P_{2}$ and describe the ideal execution of the protocol. We demonstrate via a sequence of games - where the first game is a real execution and the last game is a simulated execution - that both executions are indistinguishable. This proof methodology differs considerably to that of [Lin17] since the main differences between a real and simulated execution are due to the ciphertext $c_{k e y}$, so the indistinguishability of both executions reduces to the ind-cpa security of the underlying encryption scheme. The necessity for the properties required of HPS will thus here become apparent. We first describe an ideal execution of the protocol:

\section{Key Generation Phase}

1. Given input KeyGen $(\mathbb{G}, P, q)$, the simulator $\delta$ sends $\operatorname{KeyGen}(\mathbb{G}, P, q)$ to the functionality $\mathscr{F}_{E C D S A}$ and receives back $Q$.

2. $\mathcal{S}$ invokes $\mathcal{A}_{2}$ upon input IKeyGen $(\mathbb{G}, P, q)$ and sends (proof - receipt, 1) as $\mathcal{A}_{2}$ expects to receive from $\mathcal{F}_{\mathrm{zk}-\mathrm{com}}^{R_{D L}}$.

3. $\mathcal{S}$ receives (prove, $2, Q_{2}, x_{2}$ ) as $\mathcal{A}_{2}$ intends to send to $\mathscr{F}_{\mathrm{zk}}^{R_{D L}}$.

4. Using the extracted value $x_{2}, \delta$ verifies that $Q_{2}$ is a non zero point on the curve and that $Q_{2}=x_{2} P$. If so it computes $Q_{1} \leftarrow\left(x_{2}\right)^{-1} Q$ and sends (decom - proof, $1, Q_{1}$ ) to $\mathcal{A}_{2}$ as it expects to receive from $\mathcal{F}_{\mathrm{zk}-\mathrm{com}}^{R_{D L}}$. If not it simulates $P_{1}$ aborting and halts.

5. $\&$ samples $\mathrm{hk} \hookleftarrow \mathscr{D}_{\mathrm{hk}}$ and computes $\mathrm{hp} \leftarrow \alpha(\mathrm{hk})$. It also samples $\tilde{x}_{1} \stackrel{\$}{\leftarrow} \mathbf{Z} / q \mathbf{Z}$ and $(u, w) \in \mathrm{R}$ and computes $c_{k e y} \leftarrow \operatorname{Enc}\left((u, w) ;\left(\mathrm{hp}, \tilde{x}_{1}\right)\right)$.

6. $\delta$ sends (proof, $3,\left(\mathrm{hp}, c_{k e y}, Q_{1}\right)$ ) to $\mathcal{A}_{2}$, as $\mathcal{A}_{2}$ expects to receive from $\mathscr{F}_{\mathrm{zk}}^{R \mathrm{HPS}-\mathrm{DL}}$.

7. $\delta$ sends continue to $\mathscr{F}_{E C D S A}$ for $P_{1}$ to receive output, and stores $Q$.

\section{Signing Phase}

1. Upon input $\operatorname{Sign}(s i d, m)$, simulator $\mathcal{S}$ sends $\operatorname{Sign}(\operatorname{sid}, m)$ to $\mathscr{F}_{E C D S A}$ and receives back a signature $(r, s)$.

2. $\delta$ computes the point $R=\left(r, r_{y}\right)$ using the ECDSA verification algorithm.

3. $\mathcal{S}$ invokes $\mathcal{A}_{2}$ with input $\operatorname{ISign}(\operatorname{sid}, m)$ and sends (proof - receipt, sid $\| 1$ ) as $\mathcal{A}_{2}$ expects to receive from $\mathscr{F}_{\mathrm{zk}-\mathrm{com}}^{R_{D L}}$.

4. $\delta$ receives (prove, sid $\| 2, R_{2}, k_{2}$ ) as $\mathcal{A}_{2}$ intends to send to $\mathcal{F}_{\mathrm{zk}}^{R_{D L}}$.

5. Using the extracted value $k_{2}, \delta$ verifies that $R_{2} \neq 0_{E}$ and that $R_{2}=k_{2} P$. If so it computes $R_{1} \leftarrow k_{2}^{-1} R$ and sends (decom - proof, sid $\| 1, R_{1}$ ) to $\mathcal{A}_{2}$ as it expects to receive from $\mathcal{F}_{\mathrm{zk}-\mathrm{com}}^{R_{D L}}$. If not it simulates $P_{1}$ aborting and instructs the trusted party computing $\mathscr{F}_{E C D S A}$ to abort. 
6. $\mathcal{S}$ receives $c_{3}=(\bar{u}, \bar{e})$ from $\mathcal{A}_{2}$, which it can decrypt using hk, i.e. $\alpha \leftarrow$ Decode $\left(\frac{\bar{e}}{\mathcal{H}_{\mathrm{hk}}(\bar{u})}\right)$.

If $\alpha=k_{2}^{-1} \cdot\left(m^{\prime}+r \cdot x_{2} \cdot \tilde{x}_{1}\right) \bmod q$ then $\mathcal{S}$ sends continue to the trusted party $\mathscr{F}_{E C D S A}$, s.t. the honest party $P_{1}$ receives output. Otherwise it instructs $\mathscr{F}_{E C D S A}$ to abort.

We now describe the sequence of games. Game is $_{0}$ the real execution of the protocol, and we finish in Game 6 which is the ideal simulation described above. In the following intermediary games, only the differences in the steps performed by $\mathcal{S}$ are depicted.

\begin{tabular}{|c|c|c|}
\hline Game $_{0}$ & Game $_{1}$ & Game $_{2}$ \\
\hline$Q \leftarrow x_{1} x_{2} P$ & $Q \leftarrow x_{1} x_{2} P$ & $Q \leftarrow x_{1} x_{2} P$ \\
\hline$\vdots$ & $\vdots$ & \\
\hline $\begin{array}{l}\mathrm{hk} \leftarrow \mathscr{D}_{\mathrm{hk}} \\
\mathrm{hp} \leftarrow \alpha(\mathrm{hk})\end{array}$ & $\begin{aligned} \mathrm{hk} & \leftarrow \mathscr{D}_{\mathrm{hk}} \\
\mathrm{hp} & \leftarrow \alpha(\mathrm{hk})\end{aligned}$ & $\begin{array}{c}\mathrm{hk} \leftarrow \mathscr{D}_{\mathrm{hk}} \\
\mathrm{hp} \leftarrow \alpha(\mathrm{hk})\end{array}$ \\
\hline$c_{k e y} \leftarrow \operatorname{Enc}\left(\mathrm{hp}, x_{1}\right)$ & $\begin{array}{c}\text { Sample }(u, w) \in \mathrm{R} \\
c_{\text {keu }} \leftarrow\left(u, \mathcal{H}_{\mathrm{hk}}(u) \cdot \operatorname{Encode}\left(x_{1}\right)\right)\end{array}$ & $\begin{array}{c}\tilde{u} \stackrel{\$}{\leftarrow} X \backslash \mathcal{L} \\
c_{k e u} \leftarrow\left(\tilde{u}, \mathcal{H}_{\mathrm{hk}}(\tilde{u}) \cdot \operatorname{Encode}\left(x_{1}\right)\right)\end{array}$ \\
\hline$\vdots$ & I & ( \\
\hline$R \leftarrow k_{1} k_{2} P$ & $R \leftarrow k_{1} k_{2} P$ & $R \leftarrow k_{1} k_{2} P$ \\
\hline & $\mathrm{Game}_{3}$ & $\mathrm{Game}_{4}$ \\
\hline & $Q \leftarrow x_{1} x_{2} P$ & $Q \leftarrow x_{1} x_{2} P$ \\
\hline & $\vdots$ & \\
\hline & $\begin{array}{l}\mathrm{hk} \hookleftarrow \mathscr{D}_{\mathrm{hk}} \\
\mathrm{hp} \leftarrow \alpha(\mathrm{hk})\end{array}$ & $\begin{array}{c}\mathrm{hk} \hookleftarrow \mathscr{D}_{\mathrm{hk}} \\
\mathrm{hp} \leftarrow \alpha(\mathrm{hk})\end{array}$ \\
\hline $\begin{array}{r}\tilde{u} \\
c_{k e y} \leftarrow(\tilde{u}\end{array}$ & 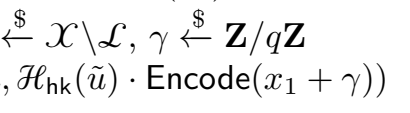 & $\begin{array}{c}\text { Sample }(u, w) \in \mathrm{R}, \gamma \stackrel{\$}{\leftarrow} \mathbf{Z} / q \mathbf{Z} \\
c_{k e y} \leftarrow\left(u, \mathcal{H}_{\mathrm{hk}}(u) \cdot \operatorname{Encode}\left(x_{1}+\gamma\right)\right)\end{array}$ \\
\hline & $R \leftarrow k_{1} k_{2} P$ & $R \leftarrow k_{1} k_{2} P$ \\
\hline & Game $_{5}$ & Game $_{6}$ \\
\hline & $Q \leftarrow x_{1} x_{2} P$ & $Q \leftarrow \mathscr{F}_{E C D S A}$ \\
\hline & $\vdots$ & $\vdots$ \\
\hline & $\begin{aligned} \mathrm{hk} & \hookleftarrow \mathscr{D}_{\mathrm{hk}} \\
\mathrm{hp} & \leftarrow \alpha(\mathrm{hk})\end{aligned}$ & $\begin{array}{c}\mathrm{hk} \hookleftarrow \mathscr{D}_{\mathrm{hk}} \\
\mathrm{hp} \leftarrow \alpha(\mathrm{hk})\end{array}$ \\
\hline & Sample $\gamma \stackrel{\$}{\leftarrow} \mathbf{Z} / q \mathbf{Z}$ & Sample $\gamma \stackrel{\$}{\leftarrow} \mathbf{Z} / q \mathbf{Z}$ \\
\hline & key $\left.\leftarrow \operatorname{Enc}\left(\mathrm{hp}, x_{1}+\gamma\right)\right)$ & $\left.c_{k e y} \leftarrow \operatorname{Enc}\left(\mathrm{hp}, x_{1}+\gamma\right)\right)$ \\
\hline & $R \leftarrow k_{1} k_{2} P$ & $R \leftarrow \dot{\mathscr{F}}_{E C D S A}$ \\
\hline
\end{tabular}

We now demonstrate that the previous games are indistinguishable from the view of $\mathcal{A}_{2}$. Intuitively, in Game $_{1}$ the simulator uses the secret hashing key hk instead of the public projection key hp to compute $c_{k e y}$. Though the values are computed differently, they are distributed identically, and are perfectly indistinguishable. Next in Game 2 we replace the first element of the ciphertext (in Game ${ }_{1}$ this is $u \in \mathcal{L}$ ) with an element $\tilde{u} \in \mathcal{X} \backslash \mathcal{L}$. By the hardness of the subset membership problem Game G $_{1}$ and $\mathrm{Game}_{2}$ are indistinguishable. Next in $\mathrm{Game}_{3}$ we multiply the second element of the ciphertext by a random element of the subgroup group $\mathscr{F}$ in which messages are encoded (or equivalently, add a random element of $\mathbf{Z} / q \mathbf{Z}$ to $x_{1}$ such that this sum will be encoded in $\left.\mathscr{F}\right)$, under the assumption that the hash proof system is $\delta_{s}$-smooth over $\mathcal{X}$ in $\mathscr{F}$, the obtained distributions of the public key and 
ciphertext (as seen by an adversary) are $\delta_{s}-$ close. So both games are indistinguishable. We then again use the hardness of the subset membership problem underlying the hash proof to hop from Game 3 to $\mathrm{Game}_{4}$, such that in the latter the first element of the ciphertext is once again in $\mathcal{L}$; and again $\mathrm{Game}_{4}$ to Game 5 are identical from an adversary's point of view since we simply use the public evaluation function of the hash function $\mathcal{H}$ instead of the private one. And finally in Game 6 we change the way $R$ and $Q$ are generated.

We denote by $E_{i}$ the probability that an algorithm interacting with the simulator in Game $\mathrm{i}_{\mathrm{i}}$ outputs 1 . Thus by demonstrating that $\left|\operatorname{Pr}\left[\mathrm{E}_{0}\right]-\operatorname{Pr}\left[\mathrm{E}_{6}\right]\right|$ is negligible, we demonstrate that - from $\mathcal{A}_{2}$ 's view, the real and ideal executions are indistinguishable.

Invalid ciphertexts: We define the notion of invalid ciphertexts as these will be useful in our game steps. A ciphertext is said to be invalid if it is of the form $\left(u^{\prime}, e^{\prime}\right):=\left(u^{\prime}, \mathcal{H}_{\mathrm{hk}}\left(u^{\prime}\right)\right.$. Encode $\left(m^{\prime}\right)$ ) where $u^{\prime} \in \mathcal{C} \backslash \mathcal{L}$. Note that one can compute such a ciphertext using the secret hashing key hk, but not the public projection key hp; that the decryption algorithm applied to $\left(u^{\prime}, e^{\prime}\right)$ with secret key hk recovers $m^{\prime}$; and that an invalid ciphertext is indistinguishable of a valid one under the hardness of the subset membership problem.

Homomorphic properties over invalid ciphertexts: It is easy to verify that homomorphic operations hold even if a ciphertext is invalid, whether this be between two invalid ciphertexts of between a valid and invalid ciphertext. This is true since the homomorphic properties we required of the hash family hold over the whole group $\mathcal{X}$ (and not only in $\mathcal{L}$ ).

$\mathrm{Game}_{0}$ to $\mathrm{Game}_{1}$. In Game $\mathrm{C}_{0}$ and in $\mathrm{Game}_{1}, Q$ and $R$ are computed in the same way. The only difference between $\mathrm{Game}_{0}$ and $\mathrm{Game}_{1}$ is the way $c_{k e y}$ is computed, namely we use the secret hashing key hk instead of the public projection key hp and the witness $w$ to compute $c_{k e y}$. Though the values are computed differently, they are distributed identically, and are perfectly indistinguishable from an adversary's point of view:

$$
\left|\operatorname{Pr}\left[E_{0}\right]-\operatorname{Pr}\left[E_{1}\right]\right|=0 .
$$

$\mathrm{Game}_{1}$ to $\mathrm{Game}_{2}$. Suppose that $\mathscr{D}$ is able to distinguish, with non negligible advantage, between the distribution generated in $\mathrm{Game}_{1}$ from that generated in $\mathrm{Game}_{2}$. Then we can devise $\hat{\mathcal{S}}$ that can use $\mathscr{D}$ to break the hard subset membership assumption, i.e., distinguish random elements of $\mathcal{L}$ from those of $X \backslash \mathcal{L}$. The input of $\hat{\mathcal{S}}$ is a hard subset membership challenge $x^{*}$ which is either an element in $\mathcal{L}$ or an element of $\mathcal{X} \backslash \mathcal{L}$. Precisely $\hat{\mathcal{S}}$ works as $\mathcal{S}$ would in $\mathrm{Game}_{1}$, interacting with the distinguisher $\mathscr{D}$ instead of $\mathcal{A}_{2}$, the only difference being that instead of sampling $(u, w) \in \mathrm{R}$ it sets $u:=x^{*}$ and computes $c_{k e y} \leftarrow\left(u, \mathcal{H}_{\mathrm{hk}}(u)\right.$. Encode $\left.\left(x_{1}\right)\right)$. When $\mathscr{D}$ returns a bit $b$ (relative to $\mathrm{Game}_{b+1}$ ), $\hat{\mathcal{S}}$ returns the same bit, where 0 represents the case $x^{*} \in \mathcal{L}$ and 1 represents the case $x^{*} \in \mathcal{X} \backslash \mathcal{L}$.

Analysis - Case $x^{*} \in \mathcal{L}:$ There exists $w \in \mathscr{W}$ such that $\left(x^{*}, w\right) \in \mathrm{R}$ and $\mathscr{H}_{\mathrm{hp}}\left(x^{*}, w\right)=$ $\mathcal{H}_{h k}\left(x^{*}\right)$. So $c_{k e y}=(u, e)$ is an encryption of $x_{1}$ as computed in Game ${ }_{1}$. Case $x^{*} \in \mathcal{X} \backslash \mathcal{L}$ : The ciphertext is $\left(x^{*}, \mathcal{H}_{\mathrm{hk}}\left(x^{*}\right) \cdot \operatorname{Encode}\left(x_{1}\right)\right)$. Which is exactly the distribution obtained in Game $_{2}$. So the advantage of $\hat{\delta}$ in breaking the hard subset membership assumption is at least that of $\mathscr{D}$ in distinguishing both games. Thus:

$$
\left|\operatorname{Pr}\left[E_{2}\right]-\operatorname{Pr}\left[E_{1}\right]\right| \leq \delta_{\mathscr{L}} .
$$


Game $_{2}$ to Game Get us denote $\tilde{x}_{1}:=x_{1}+\gamma \bmod q$. Under the assumption that the hash proof system is $\delta_{s}$-smooth over $\mathcal{X}$ in $\mathscr{F}$ (i.e. the co-domain of Encode), it holds that the distribution of $\left(x^{*}, \mathscr{H}_{\mathrm{hk}}\left(x^{*}\right) \cdot \operatorname{Encode}\left(x_{1}\right)\right)$ and of $\left(x^{*}, \mathscr{H}_{\mathrm{hk}}\left(x^{*}\right) \cdot \operatorname{Encode}\left(x_{1}\right) \cdot \operatorname{Encode}(\gamma)=\right.$ $\left.\mathcal{H}_{\mathrm{hk}}\left(x^{*}\right) \cdot \operatorname{Encode}\left(\tilde{x}_{1}\right)\right)$ for some random $\tilde{x}_{1} \in \mathbf{Z} / q \mathbf{Z}$ are $\delta_{s}-\operatorname{close}$. Thus replacing $\left(x^{*}, \mathcal{H}_{\mathrm{hk}}\left(x^{*}\right)\right.$. Encode $\left.\left(x_{1}\right)\right)$ by $\left.\left(x^{*}, \mathcal{H}_{\mathrm{hk}}\left(x^{*}\right) \cdot \operatorname{Encode}\left(\tilde{x}_{1}\right)\right)\right)$ - as is done from Game 2 to Game $\mathrm{G}_{3}$ - cannot be noticed by any PT adversary with advantage greater than $\delta_{s}$ and:

$$
\left|\operatorname{Pr}\left[\mathrm{E}_{3}\right]-\operatorname{Pr}\left[\mathrm{E}_{2}\right]\right| \leq \delta_{s}
$$

Game $_{3}$ to $\mathrm{Game}_{4}$. The change here is exactly that between Game 1 and Game 2 , thus both games are indistinguishable under the hardness of the subset membership problem on which relies the hash proof system and:

$$
\left|\operatorname{Pr}\left[\mathrm{E}_{4}\right]-\operatorname{Pr}\left[\mathrm{E}_{3}\right]\right| \leq \delta_{\mathcal{L}}
$$

Game $_{4}$ to Game $_{5}$. The change here is exactly that between Game 1 and Game 0 , thus both games are perfectly indistinguishable, even for an unbounded adversary, thus:

$$
\left|\operatorname{Pr}\left[E_{5}\right]-\operatorname{Pr}\left[E_{4}\right]\right|=0 .
$$

Game $_{5}$ to Game 6 . The only differences between Game 5 and Game 6 are the ways $Q$ and $R$ are generated. In Game $5, Q$ and $R$ derive from a Diffie-Hellman Key Exchange, which can be simulated. Moreover, since the ideal functionality $\mathscr{F}_{E C D S A}$ samples $Q$ and $R$ uniformly at random from the group $\mathbb{G}$, it holds that $x_{2}^{-1} Q$ and $x_{1} P$ have the same distribution, as do $k_{2}^{-1} R$ and $k_{1} P$. All other steps of $\mathrm{Game}_{5}$ and $\mathrm{Game}_{6}$ are identical. We conclude that, in the $\mathscr{F}_{\mathrm{zk}}, \mathscr{F}_{\mathrm{zk}-\mathrm{com}}$ hybrid model, Game ${ }_{5}$ and $\mathrm{Game}_{6}$ are identical from $\mathcal{A}_{2}$ 's view, and so:

$$
\left|\operatorname{Pr}\left[\mathrm{E}_{6}\right]-\operatorname{Pr}\left[\mathrm{E}_{5}\right]\right|=0 .
$$

Real/Ideal executions. Putting together the above probabilities, we get that:

$$
\left|\operatorname{Pr}\left[\mathrm{E}_{6}\right]-\operatorname{Pr}\left[\mathrm{E}_{0}\right]\right| \leq 2 \delta_{\mathcal{L}}+\delta_{s},
$$

and so, assuming the hardness of the subset membership problem underlying HPS, and assuming the smoothness of HPS, it holds that the real and ideal executions are computationally indistinguishable from $\mathcal{A}_{2}$ 's view, which concludes the proof of the theorem.

\section{Instantiation in Class Groups of an Imaginary Quadratic Field}

In this section, we give an instantiation of a hash proof system with the required properties in order to apply the generic construction of the previous section. For that we will use a linearly homomorphic encryption scheme modulo a prime number, denoted CL in the following, introduced in CL15] using a group with an easy Dlog subgroup, with a concrete instantiation using class groups of quadratic fields. In order to define a HPS, we use the recent results of [CLT18 that enhance the CL framework by introducing a hard subgroup membership assumption (HSM). We first give the definition of this assumption in the context of a group with an easy Dlog subgroup, then the instantiation with class groups, and then define a HPS from HSM and prove that it has the required properties to instantiate the generic construction Section 3 . 


\subsection{A Hard Subgroup Membership Assumption}

To start with, we explicitly define the generator GenGroup used in the framework of a group with an easy Dlog subgroup introduced in [CL15] and enhanced in [CLT18], with small modifications as discussed below.

Definition 4. Let GenGroup be a pair of algorithms (Gen, Solve). The Gen algorithm is a group generator which takes as inputs a parameter $\lambda$ and a prime $q$ and outputs a tuple $\left(\tilde{s}, g, f, g_{q}, \widehat{G}, G, F, G^{q}\right)$. The set $(\widehat{G}, \cdot)$ is a finite abelian group of order $q \cdot \widehat{s}$ where the bitsize of $\widehat{s}$ is a function of $\lambda$ and $\operatorname{gcd}(q, \widehat{s})=1$. The algorithm Gen only outputs an upper bound $\tilde{s}$ of $\widehat{s}$. It is also required that one can efficiently recognise valid encodings of elements in $\widehat{G}$. The set $(F, \cdot)$ is the unique cyclic subgroup of $\widehat{G}$ of order $q$, generated by $f$. The set $(G, \cdot)$ is a cyclic subgroup of $\widehat{G}$ of order $q \cdot s$ where $s$ divides $\widehat{s}$. By construction $F \subset G$, and, denoting $G^{q}:=\left\{x^{q}, x \in G\right\}$ the subgroup of order $s$ of $G$, it holds that $G=G^{q} \times F$. The algorithm Gen outputs $f, g_{q}$ and $g:=f \cdot g_{q}$ which are respective generators of $F, G^{q}$ and $G$. Moreover, the Dlog problem is easy in $F$, which means that the Solve algorithm is a deterministic polynomial time algorithm that solves the discrete logarithm problem in F:

$$
\begin{aligned}
\operatorname{Pr}\left[x=x^{\star}:\left(\tilde{s}, g, f, g_{q}, \widehat{G}, G, F, G^{q}\right) \leftarrow \operatorname{Gen}\left(1^{\lambda}, q\right), x\right. & \stackrel{\$}{\leftarrow} \mathbf{Z} / q \mathbf{Z}, X \leftarrow f^{x}, \\
& \left.x^{\star} \leftarrow \operatorname{Solve}\left(q, \tilde{s}, g, f, g_{q}, \widehat{G}, G, F, G^{q}, X\right)\right]=1 .
\end{aligned}
$$

Remark 1. In this definition, there are a few modifications compared to the definition of [CLT18]. Namely we take as input the prime $q$ instead of having Gen generating it, and we output the group $\widehat{G}$ from which the group $G$ with an easy Dlog subgroup $F$ is produced. In practice, with the concrete instantiation with class groups, this is a just a matter of rewriting: the prime $q$ was generated independently of the rest of the output in [CL15]CLT18 so it can be an input of the algorithm, and the group $\widehat{G}$ would be the class group which was implicitly defined by its discriminant. We note that it is easy to recognise valid encodings of $\widehat{G}$ while it will be not so for elements of $G \subset \widehat{G}$. This is an important difference with Paillier's encryption, and one of the reason why Lindell's $L_{P D L}$ proof does not work in our setting.

We recall here the definition of a hard subgroup membership (HSM) problem within a group with an easy Dlog subgroup as defined in CLT18. HSM is closely related to Paillier's DCR assumption. Such hard subgroup membership problems are based on a long line of assumptions on the hardness of distinguishing powers in groups. In short, DCR and HSM are essentially the same assumption but in different groups, hence there is no direct reduction between them. We emphasise that this assumption is well understood both in general, and for the specific case of class groups of quadratic fields, which we will use to instantiate GenGroup. It was first used by [CLT18 within class groups, this being said, cryptography based on class groups is now well established, and is seeing renewed interest as it allows versatile and efficient solutions such as encryption switching protocols [CIL17, inner product functional encryption [CLT18] or verifiable delay functions [BBBF18 Wes19.

In Def. 4. one has $G=F \times G^{q}$. The assumption is that it is hard to distinguish the elements of $G^{q}$ in $G$.

Definition 5 (HSM assumption). We say that GenGroup is the generator of a HSM group with easy Dlog subgroup $F$ if it holds that the HSM problem is hard even with access to the Solve algorithm. Let $\mathscr{D}\left(\right.$ resp. $\left.\mathscr{D}_{q}\right)$ be a distribution over the integers such that the distribution 
$\left\{g^{x}, x \hookleftarrow \mathscr{D}\right\}$ (resp. $\left\{g_{q}^{x}, x \hookleftarrow \mathscr{D}_{q}\right\}$ ) is at distance less than $2^{-\lambda}$ from the uniform distribution in $G$ (resp. in $G^{q}$ ). Let $\mathcal{A}$ be an adversary for the HSM problem, its advantage is defined as:

$$
\begin{gathered}
\operatorname{Adv}_{\mathcal{A}}^{\mathrm{HSM}}(\lambda)=\mid 2 \cdot \operatorname{Pr}\left[b=b^{\star}:\left(\tilde{s}, g, f, g_{q}, \widehat{G}, G, F, G^{q}\right) \leftarrow \operatorname{Gen}\left(1^{\lambda}, q\right),\right. \\
x \hookleftarrow \mathcal{D}, x^{\prime} \hookleftarrow \mathscr{D}_{q}, b \stackrel{\$}{\leftarrow}\{0,1\}, Z_{0} \leftarrow g^{x}, Z_{1} \leftarrow g_{q}^{x^{\prime}}, \\
\left.b^{\star} \leftarrow \mathcal{A}\left(q, \tilde{s}, g, f, g_{q}, \widehat{G}, G, F, G^{q}, Z_{b}, \text { Solve }(.)\right)\right]-1 \mid
\end{gathered}
$$

The HSM problem is said to be hard in $G$ if for all probabilistic polynomial time attacker $\mathcal{A}, \operatorname{Adv}_{\mathcal{A}}^{\mathrm{HSM}}(\lambda)$ is negligible.

Class groups Our instantiation makes use of class groups of orders of imaginary quadratic fields. We refer the interested reader to BH01] for background on this algebraic object and its early use in cryptography. We here briefly sketch an instantiation of algorithm GenGroup in Definition 4 , following [CL15, Fig. 2]. The formal description is given in Fig. 6 below and concrete details can be found in [CL15]. Let $q$ be a prime. We construct a fundamental discriminant $\Delta_{K}:=-q \cdot \tilde{q}$ where $\tilde{q}$ is a prime such that $q \cdot \tilde{q} \equiv-1(\bmod 4)$ and $(q / \tilde{q})=-1$. We then consider the non-maximal order of discriminant $\Delta_{q}:=q^{2} \cdot \Delta_{K}$ and its class group $\widehat{G}:=C l\left(\Delta_{q}\right)$ whose order is $h\left(\Delta_{q}\right)=q \cdot h\left(\Delta_{K}\right)$ where $h\left(\Delta_{K}\right)$ is the class number, i.e, the order of $C l\left(\Delta_{K}\right)$, the class group of fundamental discriminant $\Delta_{k}$. This number is known to satisfy the following inequality (see [Coh00, p. 295] for instance): $h\left(\Delta_{K}\right)<\frac{1}{\pi} \log \left|\Delta_{K}\right| \sqrt{\left|\Delta_{K}\right|}$ which is the bound we take for $\tilde{s}$ (a slightly better bound can be computed from the analytic class number formula).

Elements of $\widehat{G}$ are classes of ideals of the order of discriminant $\Delta_{q}$. Such classes can be represented by a unique reduced ideal. Moreover ideals can be represented using the socalled two elements representation which correspond to their basis as a lattice of dimension two. Informally, classes can be uniquely represented by two integers $(a, b), a, b<\sqrt{\left|\Delta_{q}\right|}$ and one can efficiently verify that this indeed defines an element of $\widehat{G}$ (by checking if $b^{2} \equiv \Delta_{q}$ $(\bmod 4 a))$. The arithmetic in class groups (which corresponds to reduction and composition of quadratic forms) is very efficient: the algorithms have a quasi linear time complexity using fast arithmetic (see [Coh00]).

Following [CL15, Fig. 2], we build a generator $g_{q}$ of a cyclic subgroup of $q$-th powers of $\widehat{G}$, and denote $G^{q}:=\left\langle g_{q}\right\rangle$. Then we build a generator $f$ for the subgroup $F$ of order $q$, and then set $g:=f \cdot g_{q}$ as a generator of a cyclic subgroup $G$ of $C l\left(\Delta_{q}\right)$ of order $q \cdot s$, where $s$ is unknown. Computing discrete logarithms is easy in $F$ thanks to the following facts. We denote the surjection $\bar{\varphi}_{q}: C l\left(\Delta_{q}\right) \longrightarrow C l\left(\Delta_{K}\right)$. From [CL09, Lemma 1], its kernel is cyclic of order $q$ and is generated by $f$ represented by $\left(q^{2}, q\right)$. Moreover, if $1 \leqslant m \leqslant q-1$ then, once reduced, $f^{m}$ is of the form $\left(q^{2}, L(m) q\right)$ where $L(m)$ is the odd integer in $[-q, q]$ such that $L(m) \equiv 1 / m(\bmod q)$, which gives the efficient algorithm to compute discrete logarithms in $\langle f\rangle$.

Note that following [CL15] the bit size of $q$ must have at least $\lambda$ bits, where $\lambda$ is the security parameter, which is the case for ECDSA: $q$ will be the order of the elliptic curve. The size $\eta(\lambda)$ of $\Delta_{K}$ is chosen to resist the best practical attacks, which consists in computing discrete logarithms in $C l\left(\Delta_{K}\right)$ (or equivalently the class number $h\left(\Delta_{K}\right)$ ). An index-calculus 


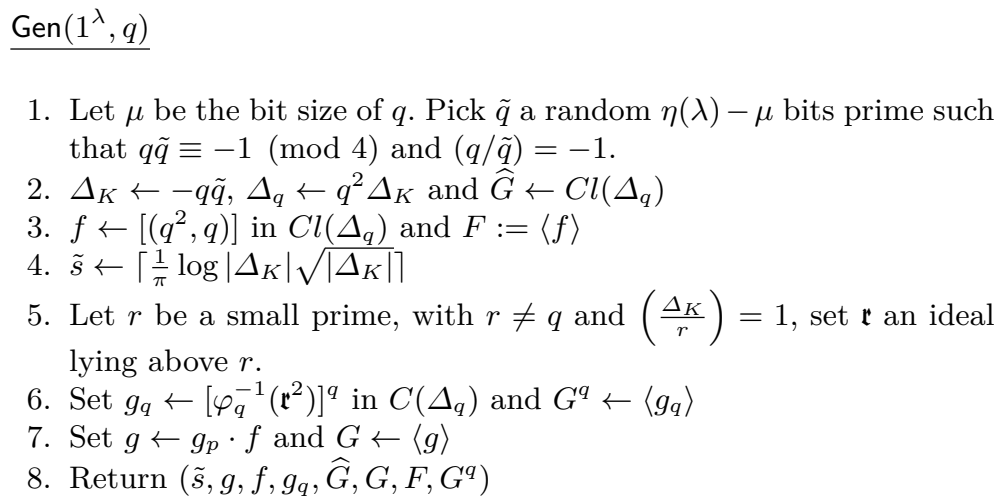

Fig. 6: Group generator Gen

method to solve the Dlog problem in a class group of imaginary quadratic field of discriminant $\Delta_{K}$ was proposed in [Jac00. It is conjectured in BJS10] that a state of the art implementation of this algorithm has complexity $\mathcal{O}\left(L_{\left|\Delta_{K}\right|}[1 / 2, o(1)]\right)$, which allows to use asymptotically shorter keys compared to protocols using classical problems that are solved in subexponential complexity $\mathcal{G}(L[1 / 3, o(1)]$ ) (see Section 5 for concrete sizes for $\eta$ ).

\subsection{A Smooth Homomorphic Hash Proof System from HSM}

We set $\mathcal{X}:=G$ and $\mathcal{L}:=G^{q}$ then $\mathcal{X} \cap \mathcal{L}=G^{q}$ and the HSM assumption states that it is hard to distinguish random elements of $G$ from those of $G^{q}$. This clearly implies the hardness of the subset membership problem, i.e., it is hard to distinguish random elements of $G \backslash G^{q}$ from those of $G^{q}$.

Let $\mathscr{D}$ be a distribution over the integers such that the distribution $\left\{g^{w}, w \hookleftarrow \mathscr{D}\right\}$ is at distance $\delta_{\mathscr{D}} \leq 2^{-\lambda}$ of the uniform distribution in $G$.

Associated projective hash family. Let PHF be the projective hash family associated to the above subset membership problem, the description of which specifies:

- A hash key space $K:=\mathbf{Z}$.

- A keyed hash function, with input and output domain $G$, s.t., for hk $\hookleftarrow \mathscr{D}$, and for $x \in G, \mathcal{H}_{\mathrm{hk}}(x):=x^{\mathrm{hk}}$.

- An auxiliary function $\alpha: K \mapsto G^{q}$ such that for hk $\in K, \alpha(\mathrm{hk}):=\mathscr{H}_{\mathrm{hk}}\left(g_{q}\right)=g_{q}^{\mathrm{hk}}$. Notice that for a hash key hk, and for $x \in G^{q}$, the knowledge of $\alpha(\mathrm{hk})$ completely determines the value $\mathscr{H}_{\mathrm{hk}}(x)$.

- An efficient public evaluation function, such that, for $x \in G^{q}$ with witness $w$ such that $x=g_{q}^{w}$ one can efficiently compute $\mathcal{H}_{\mathrm{hk}}(x)=\alpha(\mathrm{hk})^{w}=x^{\mathrm{hk}}$ knowing only the value of the auxiliary function $\alpha(\mathrm{hk})$ (but not hk).

Lemma 1 (Smoothness). The projective hash family PHF is $\delta_{s}$-smooth over $G$ in $F$, with $\delta_{s} \leqslant 2 \delta_{\mathscr{D}}$, i.e., for any $x \in G \backslash G^{q}, \pi \leftarrow f^{\gamma} \in F \subset G$ where $\gamma \stackrel{\$}{\leftarrow} \mathbf{Z} / q \mathbf{Z}$ and $k \hookleftarrow \mathscr{D}$, the distributions $\mathscr{D}_{1}:=\left\{x, g_{q}^{k}, \pi \cdot x^{k}\right\}$ and $\mathscr{D}_{2}:=\left\{x, g_{q}^{k}, x^{k}\right\}$ are less than $2 \delta_{\mathscr{D}}$-close. 
Proof. For $x \in G \backslash G^{q}$, there exist $a \in \mathbf{Z} / s \mathbf{Z}$ and $b \in(\mathbf{Z} / q \mathbf{Z})^{*}$ such that $x=g_{q}^{a} f^{b}$. Thus we can write $\mathscr{D}_{1}=\left\{g_{q}^{a} f^{b}, g_{q}^{k}, g_{q}^{a \cdot k} f^{b \cdot k+\gamma}\right\}$ and $\mathscr{D}_{2}=\left\{g_{q}^{a} f^{b}, g_{q}^{k}, g_{q}^{a \cdot k} f^{b \cdot k}\right\}$. It remains to study the statistical distance of the third coordinates of the two distributions, given the two first coordinates, i.e, if $(a \bmod s),(b \bmod q)$, and $(k \bmod s)$ are fixed. This is the statistical between $X:=b \cdot k+\gamma$ and $Y:=b \cdot k$ in $\mathbf{Z} / q \mathbf{Z}$. Since $\gamma$ is uniform in $\mathbf{Z} / q \mathbf{Z}, X$ is the uniform distribution. As $\mathscr{D}$ is by definition at statistical distance $\delta_{\mathscr{D}}$ from the uniform distribution modulo $q \cdot s$, and $\operatorname{gcd}(q, s)=1$, one can prove (cf. Lemma 1 in Appendix II that even knowing $(k \bmod s)$, the distribution of $(k \bmod q)$ is at distance less than $2 \delta_{\mathscr{D}}$ from the uniform distribution over $\mathbf{Z} / q \mathbf{Z}$. As a result, the distance between $X$ and $Y$ is bounded by $2 \delta_{\mathfrak{D}}$, which concludes the proof.

Linearly homomorphic. For all hk $\in \mathbf{Z}$, and $u_{1}, u_{2} \in G, \mathcal{H}_{\mathrm{hk}}\left(u_{1}\right) \cdot \mathcal{H}_{\mathrm{hk}}\left(u_{2}\right)=u_{1}^{\mathrm{hk}} \cdot u_{2}^{\mathrm{hk}}=$ $\left(u_{1} \cdot u_{2}\right)^{\mathrm{hk}}=\mathscr{H}_{\mathrm{hk}}\left(u_{1} \cdot u_{2}\right)$. Thus $\mathscr{H}_{\mathrm{hk}}$ is a homomorphism for each hk.

Resulting encryption scheme. A direct application of Subsection 3.3 using the above HPS results in the encryption scheme called HSM-CL in [CLT18, which is linearly homomorphic modulo $q$ and ind - cpa under the HSM assumption. We describe this scheme in Fig. 7 for completeness. Note that here the secret key $x$ (and the randomness $r$ ) is drawn with a distribution $\mathscr{D}_{q}$ such that $\left\{g_{q}^{x}, x \hookleftarrow \mathscr{D}_{q}\right\}$ is at distance less than $2^{-\lambda}$ from the uniform distribution in $G^{q}$, this does not change the view of the attacker. Let $S:=2^{\lambda-2} \cdot \tilde{s}$. In practice, we will use for $\mathscr{D}_{q}$ the uniform distribution on $\{0, \ldots, S\}$.

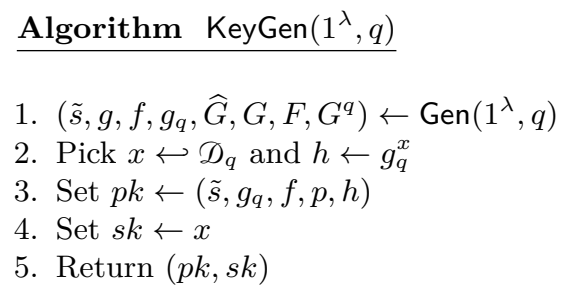

\author{
Algorithm $\operatorname{Enc}(p k, m)$ \\ 1. Pick $r \hookleftarrow \mathscr{D}_{q}$ \\ 2. Return $\left(g_{q}^{r}, f^{m} h^{r}\right)$ \\ Algorithm $\operatorname{Dec}\left(s k,\left(c_{1}, c_{2}\right)\right)$ \\ 1. Compute $M \leftarrow c_{2} / c_{1}^{x}$ \\ 2. Return Solve $(M)$
}

Fig. 7: Description of the HSM-CL encryption scheme

\subsection{A zero-knowledge proof for $R_{\mathrm{CL}-\mathrm{DL}}$}

We describe here the ZKPoK for $R_{\mathrm{HPS}-\mathrm{DL}}$ used for our instantiation with the encryption scheme of Fig. 7 and denote it $R_{\mathrm{CL}-\mathrm{DL}}$. It relies on the Schnorr-like GPS (statistically) zero-knowledge identification scheme [GPS06] that we turn into a zero-knowledge proof of knowledge of the randomness used for encryption and of the discrete logarithm of an element on an elliptic curve, using a binary challenge. This proof is partly performed in a group of unknown order.

We denote $c_{k e y}:=\left(c_{1}, c_{2}\right)$. If $c_{k e y}$ is a valid encryption of $x_{1}$ under public key pk it holds that $c_{k e y}=\left(g_{q}^{r}, f^{x_{1}} \mathrm{pk}^{r}\right)$ for some $r \in\{0, \ldots, S\}$. The protocol $R_{\mathrm{CL}-\mathrm{DL}}$ provides a ZKPoK for the following relation: 


\begin{tabular}{|c|c|c|}
\hline Input : $\left(r, x_{1}\right)$ and $\left(\mathrm{pk}, c_{1}, c_{2}, Q, P\right)$ & & Input : $\left(\mathrm{pk}, c_{1}, c_{2}, Q, P\right)$ \\
\hline \multicolumn{3}{|l|}{ Repeat $\ell$ times } \\
\hline \multicolumn{3}{|l|}{$r_{1} \stackrel{\$}{\leftarrow}\left[0, A\left[; r_{2} \stackrel{\$}{\leftarrow} \mathbf{Z} / q \mathbf{Z}\right.\right.$} \\
\hline \multirow{2}{*}{$t_{1} \leftarrow \mathrm{pk}^{r_{1}} f^{r_{2}} ; t_{2} \leftarrow r_{2} P ; t_{3} \leftarrow g_{q}^{r_{1}}$} & $t_{1}, t_{2}, t_{3}$ & \\
\hline & $k$ & $k \stackrel{\$}{\leftarrow}\{0,1\}$ \\
\hline \multicolumn{3}{|l|}{$u_{1} \leftarrow r_{1}+k r$ in $\mathbf{Z}$} \\
\hline$u_{2} \leftarrow r_{2}+k x_{1} \bmod q$ & & $\begin{array}{l}\text { Check } u_{1} \in[0, A+S[ \\
t_{1} \cdot c_{2}^{k}=\mathrm{pk}^{u_{1}} \cdot f^{u_{2}} \\
t_{2}+[k] Q=\left[u_{2}\right] P \\
t_{3} \cdot c_{1}^{k}=g_{q}^{u_{1}}\end{array}$ \\
\hline
\end{tabular}

Fig. 8: The zero-knowledge proof of knowledge $R_{\mathrm{CL}-\mathrm{DL}}$

$$
R_{\mathrm{CL}-\mathrm{DL}}:=\left\{\left(\mathrm{pk},\left(c_{1}, c_{2}\right), Q_{1}\right) ;\left(x_{1}, r\right) \mid c_{1}=g_{q}^{r} \wedge c_{2}=f^{x_{1}} \mathrm{pk}^{r} \wedge Q_{1}=x_{1} G\right\}
$$

The following theorem, whose proof is given in Appendix III states the security of the zero-knowledge proof of knowledge $R_{\mathrm{CL}-\mathrm{DL}}$.

Theorem 2. The protocol described in Figure 8 is a statistical zero-knowledge proof of knowledge with soundness $2^{-\ell}$, as long as $\ell$ is polynomial and $\ell S / A$ is negligible, where $A$ is a positive integer.

\subsection{Two-Party Distributed ECDSA Protocol from HSM}

The protocol results from a direct application of Subsection 3.5 using the HPS defined in Subsection 4.2 an the $R_{\mathrm{CL}-\mathrm{DL}}$ proof of the previous subsection. Therefore we defer the detailed protocol to Appendix $\mathrm{V}$, and simply state the following theorem.

Theorem 3. Assuming GenGroup is the generator of a HSM group with easy Dlog subgroup $F$, then the protocol of Appendix $V$ securely computes $\mathscr{F}_{E C D S A}$ in the $\left(\mathscr{F}_{\mathrm{zk}}, \mathscr{F}_{\mathrm{com}-\mathrm{zk}}\right)$-hybrid model in the presence of a malicious static adversary (under the ideal/real definition).

\section{Implementation and Efficiency Comparisons}

In this section we compare an implementation of our protocol with Lindell's protocol of [Lin17]. For fair comparison, we implement both protocols with the Pari C Library ([ $[$ PAR18] $]$, as this library handles arithmetic in class groups, $\mathbf{Z} / n \mathbf{Z}$ and elliptic curves. In particular, in this library, exponentiations in $\mathbf{Z} / n \mathbf{Z}$ and in class groups both use the same sliding window method. The running times are measured on a single core of an Intel(R) Core(TM) i7-7700 CPU @ 3.60GHz (even if key generation can easily be parallelized). We do not implement commitments (this does not bias the comparison as they appear with equal weight in both schemes), and we only measure computation time and do not include communication (again this is fair as communication is similar). 
As in [Lin17, we ran our implementation on the standard NIST curves P-256, P-384 and P-521, corresponding to levels of security 128, 192 and 256. For the encryption scheme, we start with a 112 bit security, as in [Lin17, but also study the case where its level of security matches the security of the elliptic curves.

Again as in [Lin17, we fixed the number of rounds in zero knowledge proofs to reach a statistical soundness error of $2^{-40}$. For the distributions we also set the parameters to get statistical error of $2^{-40}$. The zero knowledge proofs for $R_{D L}$ are implemented with the Schnorr protocol.

In the following, we review the theoretical complexity and experimental results of both schemes, before comparing them. In terms of theoretical complexity, exponentiations in the encryption schemes dominate the computation as elliptic curve operations are much cheaper. Thus, we only count these exponentiations; we will see this results in an accurate prediction of experimental timings.

\subsection{Lindell's Protocol with Paillier's Encryption Scheme}

The key generation uses on average 360 Paillier exponentiations (of the form $r^{N} \bmod N^{2}$ ) but not all of them are full exponentiations. The signing phase uses only 2 Paillier exponentiations.

The timings corresponds to the mean of several experiments (30 to 1000 depending the security level). The timings are quite stable other than the generation of the RSA modulus in the key generation. We use standard RSA integers (i.e., not strong prime factors) as this would be too slow for high security levels. For example, for 256 bits security (15360 bits modulus), the generation of the modulus takes 95 seconds (mean of 30 experiments) with a standard deviation of 56s. For the rest of the protocol the experimental timings are roughly equal to the number of exponentiations multiplied by the cost of one exponentiation.

The result are summarized in Fig. 9a Timings are given in milliseconds and sizes in bits. The columns corresponds to the elliptic curve used for ECDSA, the security parameter in bits for the encryption scheme, the corresponding modulus bit size, the timings of one Paillier exponentiation, of the key generation and of the signing phase and the total communication in bits for two phases. Modulus sizes are set according to the NIST recommendations.

Note that for the first line, we use a 2048 bits modulus as in Lin17 and we obtain a similar experimental result.

\subsection{Our Protocol with HSM-CL Encryption Scheme}

The key generation uses a total of 160 class group exponentiations (of the form $g_{q}^{r}$ in the class group of discriminant $\Delta_{q}=-q^{3} \cdot \tilde{q}$ ). This corresponds to the 40 rounds of the $R_{\mathrm{CL}-\mathrm{DL}}$ zero-knowledge proof of knowledge of Fig. 8 Note that exponentiations in $\langle f\rangle$ are almost free as seen in Subsection 4.1. Signing uses 3 class group exponentiations (one encryption and one decryption).

We use the same number of experiments as for Lindell's protocol. Here timings are very stable. Indeed during key generation, we only compute the public key $h \leftarrow g_{q}^{x}$ with one exponentiation, as the output of Gen (mainly the discriminant $\Delta_{q}$ of the class group and the generator $g_{q}$ ) is a common public parameter that only depends on the cardinality $q$ of the elliptic curve. As a result this can be considered as an input of the protocol, as the same group can be used by all users. Moreover doing this does not change the global result of the comparison with Lindell's protocol: the running time of Gen is dominated by the generation 


\begin{tabular}{|l|c|c|c|c|c|c|c|}
\hline Curve & Sec. Param. & Modulus & Expo. (ms) & Keygen (ms) & Signing (ms) & Keygen (b) & Signing (b) \\
\hline P-256 & 112 & 2048 & $\mathbf{7}$ & $\mathbf{2 ~ 1 3 3}$ & $\mathbf{2 0}$ & 881901 & 5636 \\
\hline P-256 & 128 & 3072 & $\mathbf{2 2}$ & $\mathbf{6 3 4 0}$ & $\mathbf{4 9}$ & 1317101 & 7684 \\
\hline P-384 & 192 & 7680 & 214 & 65986 & $\mathbf{4 3 7}$ & 3280429 & 17668 \\
\hline P-521 & 256 & 15360 & 1196 & 429965 & 2415 & 6549402 & 33832 \\
\hline
\end{tabular}

(a) Lindell's Protocol with Paillier

\begin{tabular}{|l|c|c|c|c|c|c|c|}
\hline Curve & Sec. Param. & Discriminant & Expo. $(\mathrm{ms})$ & Keygen $(\mathrm{ms})$ & Signing $(\mathrm{ms})$ & Keygen $(\mathrm{b})$ & Signing (b) \\
\hline P-256 & 112 & 1348 & 32 & 5521 & 101 & $\mathbf{1 7 8 6 6 8}$ & $\mathbf{4 7 4 8}$ \\
\hline P-256 & 128 & 1827 & 55 & 9350 & 170 & $\mathbf{2 2 7 5 2 6}$ & $\mathbf{5 7 0 6}$ \\
\hline P-384 & 192 & 3598 & $\mathbf{2 1 2}$ & $\mathbf{3 5 4 9 1}$ & 649 & $\mathbf{4 2 7 1 1 2}$ & $\mathbf{1 0 2 7 2}$ \\
\hline P-521 & 256 & 5971 & $\mathbf{6 2 3}$ & $\mathbf{1 0 3 0 9 5}$ & $\mathbf{1 8 8 8}$ & $\mathbf{6 8 8 4 9 8}$ & $\mathbf{1 6 0 7 8}$ \\
\hline
\end{tabular}

(b) Our Protocol with HSM-CL

Fig. 9: Experimental results (timings in ms, sizes in bits)

of $\tilde{q}$, a prime of size much smaller than the factor of the RSA modulus. So even if we add this running time in the Keygen column, this does not affect the results of our comparisons for any of the considered security levels.

The results are summarized in Fig. 9b. Timings are given in milliseconds and sizes in bits. The columns correspond to the elliptic curve used for ECDSA, the security parameter in bits for the encryption scheme, the corresponding fundamental discriminant $\Delta_{K}=-q \cdot \tilde{q}$ bit size, the timings of one class group exponentiation, of the key generation and of the signing phase and the total communication in bits for two phases. The discriminant sizes are chosen according to [BJS10].

\subsection{Comparison}

Figure 9 shows that Lindell's protocol is faster for both key generation and signing for standard security levels for the encryption scheme (112 and 128 bits of security) while our solution remains of the same order of magnitude. However for high security levels, our solution becomes faster (in terms of key generation from a 192-bits security level and for both key generation and signing from a 256-bits security level).

In terms of communications, our solution outperforms the scheme of Lindell at all level of security by a factor 5 to 10 for Keygen. For Signing, we gain $15 \%$ for basic security to a factor 2 at 256-bits security level. In terms of rounds, our protocol uses 126 rounds for Keygen and Lindell's protocol uses 175 rounds, so we get a $28 \%$ gain. Both protocol use 7 rounds for Signing.

This situation can be explained by the following facts. Firstly we use less than half the number of exponentiations in the key generation as we do not need a range proof: our message space is $\mathbf{Z} / q \mathbf{Z}$ as the CL encryption scheme is homomorphic modulo a prime. Secondly, with class groups of quadratic fields we can use lower parameters than with $\mathbf{Z} / n \mathbf{Z}$ (as shown in the introduction, the best algorithm against the discrete logarithm problem in class groups has complexity $\mathcal{O}(L[1 / 2, o(1)])$ compared to an $\mathcal{O}(L[1 / 3, o(1)])$ for factoring). However, the group law is more complex in class groups. By comparing the Expo. time 
columns in the tables, we see that exponentiations in class groups are cheaper from the 192 bits level. So even if we use half as many exponentiations, the key generation for our solution only takes less time from that level (while being of the same order of magnitude below this level). For signing, we increase the cost by one exponentiation due to the Elgamal structure of the CL encryption scheme. However, one can note that we can pre process this encryption by computing $\left(g_{q}^{\tau}, h^{\tau}\right)$ in an offline phase and computing $c_{1} \leftarrow\left(g_{q}^{\tau}, h^{\tau} f^{k_{2}^{-1} m^{\prime}}\right)$ which results in only one multiplication in the online phase (cf. Appendix V). As a result we will have only one exponentiation in the online signing for the decryption operation. The same holds for Lindell's protocol with Paillier. Using that both protocols take the same time for signing at the 192 bits level.

Increasing the number of rounds to obtain a $2^{-60}$ soundness error. This impacts only KeyGen, where the Lin17] scheme and ours both use 40 iterations of ZK proofs to achieve a $2^{-40}$ soundness error. Lindell's protocol performs 9 exponentiations per iteration while ours performs 4 . All timings will thus be multiplied by $3 / 2$ to achieve a $2^{-60}$ soundness error, and indeed this is what we observe in practice. Complexity is linear in the number of iterations and the ratio between our timings and those of [Lin17] remains constant.

\section{Instantiation of our Generic Construction Using DCR}

As stated at the end of the introduction, we can instantiate the generic construction of Section 3 with the hash proof system built upon Paillier's decision composite residuosity assumption (DCR). This yields the Elgamal Paillier encryption scheme of [CS03] that closely resembles the HSM-CL Encryption scheme. However, the message space is $\mathbf{Z} / n \mathbf{Z}$ as in Lindell's protocol, so in addition to the $R_{\text {HPS }}$ DL proof, $P_{1}$ has to prove that $x_{1}$ is an element of $\mathbf{Z}_{q}$ with a range proof. Moreover, this encryption scheme uses two exponentiations instead of one for Paillier. This being said a gain arrises from the fact that following the techniques

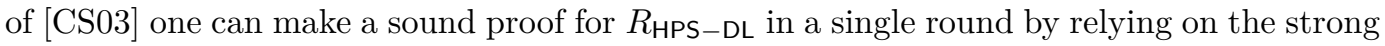
RSA assumption. This means that one should use safe primes that can be very costly to generate at high security level. However, for 112 and 128 bits of security this should give a competitive solution compared to Lindell's with a simulation based security relying on the hardness of classical problems, the DCR and the strong RSA assumptions.

\section{Conclusion}

Inspired by Lindell's scheme, we have provided the first generic construction for two-party ECDSA signing from hash proof systems which are homomorphic modulo a prime number. Theoretically, our construction allows for a simulation-based proof of security that is both tight and requires no artificial interactive assumptions, due to the structure of the underlying semantically secure homomorphic encryption schemes. Practically, we provide a detailed instantiation, and C implementation, from class groups of imaginary quadratic fields using the CL framework. This yields a better performance than Lindell's Paillier-based scheme for high levels of security, and same order of magnitude for standard levels. Our solution becomes faster than Lindell's from 192-bits of security upwards. Improvements could come from advances in ideal arithmetic in imaginary quadratic fields (see [MJJS10 for instance). Recent proposals of verifiable delay functions based on class groups should also motivate research in this area (for example the Chia Network [?] has opened a competition for this). 
Moreover, the bottleneck of our instantiation is the use of binary challenges in a zero knowledge proof of knowledge, used during key generation, in order to cope with the fact we are working in a cyclic subgroup of a group of unknown order and that we can not check that elements belong to the subgroup. There have been many proposals to deal with generalized Schnorr proofs in groups of unknown order (see for instance the framework of [CKY09] using safeguard groups, or [TW12]). For the case of subgroups of $(\mathbf{Z} / n \mathbf{Z})^{\times}$, efficient solutions for this type of proofs enlarge the challenge space, and rely on variants of the strong RSA assumption. For class groups, there have been informal proposals (see DF02 for instance). However computing square roots or finding elements of order 2 can be done efficiently in class groups knowing the factorization of the discriminant (which is public in our case). Moreover, as suggested in [BBF18], there may be other approaches to find low order elements in class groups. Advances in our understanding of class groups would lead to substantial efficiency improvements in several areas of cryptography.

Last but not least, our work focuses on the two party case. We believe that the ideas of our generic construction will lead to improvements in the general case of threshold ECDSA signatures. We leave this for future work.

Acknowledgements: The authors would like to thank Benoit Libert for fruitful discussions. This work was supported by the Universita' degli Studi di Catania,"Piano della Ricerca 2016/2018 Linea di intervento 2", and the French ANR ALAMBIC project (ANR-16-CE39$0006)$.

\section{References}

BBBF18. D. Boneh, J. Bonneau, B. Bünz, and B. Fisch. Verifiable delay functions. In CRYPTO 2018, Part I, LNCS 10991. Springer, 2018.

BBF18. D. Boneh, B. Bünz, and B. Fisch. A survey of two verifiable delay functions. Cryptology ePrint Archive, Report 2018/712, 2018. https://eprint.iacr.org/2018/712.

BH01. J. Buchmann and S. Hamdy. A survey on iq cryptography. In Proceedings of Public Key Cryptography and Computational Number Theory, 2001.

BH03. M. L. Bauer and S. Hamdy. On class group computations using the number field sieve. In ASIACRYPT 2003, LNCS 2894. Springer, 2003.

BJS10. J.-F. Biasse, M. J. Jacobson, and A. K. Silvester. Security estimates for quadratic field based cryptosystems. In ACISP 10, LNCS 6168. Springer, 2010.

Boy86. C. Boyd. Digital multisignature. Cryptography and Coding, 1986.

CH89. R. A. Croft and S. P. Harris. Public-key cryptography and reusable shared secret. Cryptography and Coding, 1989.

CIL17. G. Castagnos, L. Imbert, and F. Laguillaumie. Encryption switching protocols revisited: Switching modulo p. In CRYPTO 2017, Part I, LNCS 10401. Springer, 2017.

CKY09. J. Camenisch, A. Kiayias, and M. Yung. On the portability of generalized Schnorr proofs. In EUROCRYPT 2009, LNCS 5479. Springer, 2009.

CL09. G. Castagnos and F. Laguillaumie. On the security of cryptosystems with quadratic decryption: The nicest cryptanalysis. In EUROCRYPT 2009, LNCS 5479. Springer, 2009.

CL15. G. Castagnos and F. Laguillaumie. Linearly homomorphic encryption from DDH. In CT-RSA 2015, LNCS 9048. Springer, 2015.

CLT18. G. Castagnos, F. Laguillaumie, and I. Tucker. Practical fully secure unrestricted inner product functional encryption modulo p. In ASIACRYPT 2018, Part II, LNCS 11273. Springer, 2018.

Coh00. H. Cohen. A course in computational algebraic number theory. Springer-Verlag, 2000. 
CS98. R. Cramer and V. Shoup. A practical public key cryptosystem provably secure against adaptive chosen ciphertext attack. In CRYPTO'98, LNCS 1462. Springer, 1998.

CS02. R. Cramer and V. Shoup. Universal hash proofs and a paradigm for adaptive chosen ciphertext secure public-key encryption. In EUROCRYPT 2002, LNCS 2332. Springer, 2002.

CS03. J. Camenisch and V. Shoup. Practical verifiable encryption and decryption of discrete logarithms. In CRYPTO 2003, LNCS 2729. Springer, 2003.

Des88. Y. Desmedt. Society and group oriented cryptography: A new concept. In CRYPTO'87, LNCS 293. Springer, 1988.

DF90. Y. Desmedt and Y. Frankel. Threshold cryptosystems. In CRYPTO'89, LNCS 435. Springer, 1990.

DF02. I. Damgård and E. Fujisaki. A statistically-hiding integer commitment scheme based on groups with hidden order. In ASIACRYPT 2002, LNCS 2501. Springer, 2002.

DKLs18. J. Doerner, Y. Kondi, E. Lee, and a. shelat. Secure two-party threshold ECDSA from ECDSA assumptions. In 2018 IEEE Symposium on Security and Privacy, p. 980-997. IEEE Computer Society Press, 2018.

DKLs19. J. Doerner, Y. Kondi, E. Lee, and a. shelat. Threshold ECDSA from ECDSA assumptions: The multiparty case. In 2019 IEEE Symposium on Security and Privacy, pages 980-997. IEEE Computer Society Press, 2019.

GG18. R. Gennaro and S. Goldfeder. Fast multiparty threshold ECDSA with fast trustless setup. In ACM CCS 18. ACM Press, 2018.

GGN16. R. Gennaro, S. Goldfeder, and A. Narayanan. Threshold-optimal DSA/ECDSA signatures and an application to bitcoin wallet security. In ACNS 16, LNCS 9696. Springer, 2016.

GJKR96. R. Gennaro, S. Jarecki, H. Krawczyk, and T. Rabin. Robust threshold DSS signatures. In EUROCRYPT'96, LNCS 1070. Springer, 1996.

GMR89. S. Goldwasser, S. Micali, and C. Rackoff. The knowledge complexity of interactive proof systems. SIAM Journal on Computing, 1989.

Gol01. O. Goldreich. Foundations of Cryptography: Basic Tools. Cambridge University Press, Cambridge, UK, 2001.

GPS06. M. Girault, G. Poupard, and J. Stern. On the fly authentication and signature schemes based on groups of unknown order. Journal of Cryptology, 2006.

HL10. C. Hazay and Y. Lindell. Efficient Secure Two-Party Protocols: Techniques and Constructions. Springer-Verlag, 1st edition, 2010.

HO09. B. Hemenway and R. Ostrovsky. Lossy trapdoor functions from smooth homomorphic hash proof systems. Electronic Colloquium on Computational Complexity (ECCC), 16:127, 01 2009.

IMJJS10. L. Imbert, J. Michael J. Jacobson, and A. Schmidt. Fast ideal cubing in imaginary quadratic number and function fields. Advances in Mathematics of Communications, 2010.

Jac00. M. J. Jacobson Jr. Computing discrete logarithms in quadratic orders. Journal of Cryptology, 2000.

Lin16. Y. Lindell. How to simulate it - A tutorial on the simulation proof technique. Cryptology ePrint Archive, Report 2016/046, 2016. http://eprint.iacr.org/2016/046

Lin17. Y. Lindell. Fast secure two-party ECDSA signing. In CRYPTO 2017, Part II, LNCS 10402. Springer, 2017.

LN18. Y. Lindell and A. Nof. Fast secure multiparty ECDSA with practical distributed key generation and applications to cryptocurrency custody. In ACM CCS 2018, pages 1837-1854. ACM Press, October 2018.

MR04. P. D. MacKenzie and M. K. Reiter. Two-party generation of DSA signatures. Int. J. Inf. Sec., 2004.

PAR18. PARI Group, Univ. Bordeaux. PARI/GP version 2.11.1, 2018. available from http: //pari.math.u-bordeaux.fr/

Sch91. C.-P. Schnorr. Efficient signature generation by smart cards. Journal of Cryptology, 1991.

Sep. Sepior. http://www.sepior.com 
Ser. I. D. P. Services. https://security.intuit.com/

SG98. V. Shoup and R. Gennaro. Securing threshold cryptosystems against chosen ciphertext attack. In EUROCRYPT'98, LNCS 1403. Springer, 1998.

Sho00. V. Shoup. Practical threshold signatures. In EUROCRYPT 2000, LNCS 1807. Springer, 2000 .

TW12. B. Terelius and D. Wikström. Efficiency limitations of S-protocols for group homomorphisms revisited. In SCN 12, LNCS 7485. Springer, 2012.

Unb. Unboundtech. https://www.unboundtech.com/.

Van92. S. Vanstone. Responses to nist's proposal. Communications of the ACM, 1992. (communicated by John Anderson).

Wes19. B. Wesolowski. Efficient verifiable delay functions. In Advances in Cryptology - EUROCRYPT 2019, pages 379-407, Cham, 2019. Springer International Publishing. 


\section{Auxiliary Supporting Material}

\section{A brief definition of interactive zero-knowledge proofs}

A zero-knowledge proof system $(P, V)$ for a language $\mathcal{L}$ is an interactive protocol between two probabilistic algorithms: a prover $P$ and a polynomial-time verifier $V$. Informally $\mathrm{P}-$ who detains a witness for a given statement - must convince $V$ that the statement is true without revealing anything other than the truth of this statement to $V$.

Specifically, if $\mathcal{L}$ is a language, $x \in \mathcal{L}$ is a true statement while $x \notin \mathscr{L}$ is a false statement; and $1 \leftarrow(P, V)(x)$ (resp. $0 \leftarrow(P, V)(x)$ ) denotes the case when $V$ interacting with $P$ accepts (resp. rejects) the proof, the following properties must hold:

- Completeness: for any $x \in \mathcal{L}$ :

$$
\operatorname{Pr}[1 \leftarrow(P, V)(x)]>1 / 2
$$

- Soundness: for any prover $P^{*}$ and for any $x \notin \mathcal{L}$ :

$$
\operatorname{Pr}\left[0 \leftarrow\left(P^{*}, V\right)(x)\right]>1 / 2
$$

- Zero-knowledge: for every probabilistic polynomial time verifier $V^{*}$, there exists a probabilistic simulator Sim running in expected polynomial time such that for every $x \in \mathcal{L}$,

$$
\left(P, V^{*}\right)(x) \equiv \operatorname{Sim}(x) .
$$

$(P, V)(x)$ is a random variable representing the output of $V$ at the end of an interaction with $P$, then the zero-knowledge property holds if for any probabilistic polynomial time $V^{*}$, the output of $V^{*}$ after an interaction with $P$ is the same one of the simulator.

For a full explanation on this model see Gol01 for interactive proofs and GMR89 for zero-knowledge.

\section{A technical Lemma on Distributions}

Lemma 1. Let $X$ be a discrete random variable at statistical distance $\epsilon$ from the uniform distribution over $\mathbf{Z} / a b \mathbf{Z}$ for positive integers $a$ and $b$ such that $\operatorname{gcd}(a, b)=1$. And let $X_{a}$ $\left(\right.$ resp. $\left.X_{b}\right)$ be the random variable defined as $X_{a}:=X \bmod a\left(r e s p . X_{b}:=X \bmod b\right)$. Then the random variables $X_{a}$ and $X_{b}$ are less than $\epsilon$ close to the uniform distributions in $\mathbf{Z} / a \mathbf{Z}$ and $\mathbf{Z} / b \mathbf{Z}$ respectively. Moreover, even knowing $X_{b}, X_{a}$ remains at statistical distance less than $2 \epsilon$ of the uniform distribution in $\mathbf{Z} / a \mathbf{Z}$ (and vice versa).

Proof. Let $C$ be an algorithm which takes as input a tuple $(a, b, x) \in \mathbf{N}^{2} \times \mathbf{Z} / a b \mathbf{Z}$, which can either be a sample of the distribution:

$$
u:=\{a, b, x \mid \operatorname{gcd}(a, b)=1 \wedge x \stackrel{\$}{\leftarrow} \mathbf{Z} / a b \mathbf{Z}\}
$$

or a sample of:

$$
\mathcal{V}:=\{a, b, x \mid \operatorname{gcd}(a, b)=1 \wedge x \hookleftarrow \mathscr{D}\},
$$


where $\mathscr{D}$ is a distribution at statistical distance $\epsilon$ of the uniform distribution over $\mathbf{Z} / a b \mathbf{Z}$, and outputs a bit. Since distributions $U$ and $V$ are at statistical distance $\epsilon$, for any such algorithm $C$, it holds that:

$$
|\operatorname{Pr}[C(u) \rightarrow 1]-\operatorname{Pr}[\mathcal{C}(\mathcal{V}) \rightarrow 1]| \leq \epsilon .
$$

We further denote $U_{\mathcal{A}}:=\left\{a, b, x_{b}, x_{a} \mid(a, b, x) \hookleftarrow v ; x_{b} \leftarrow x \bmod b ; x_{a} \stackrel{\$}{\leftarrow} / a \mathbf{Z}\right\}$ and $\mathcal{V}_{\mathcal{A}}:=\left\{a, b, x_{b}, x_{a} \mid(a, b, x) \hookleftarrow \mathcal{V} ; x_{b} \leftarrow x \bmod b ; x_{a} \leftarrow x \bmod a\right\}$.

Consider any algorithm $\mathcal{A}$ which takes as input a sample $\left(a, b, x_{b}, x_{a}^{*}\right)$ of either $U_{\mathcal{A}}$ or $\mathcal{V}_{A}$, and outputs a bit $\beta^{\prime}$. $\mathcal{A}$ 's goal is to tell whether $x_{a}^{*}$ is sampled uniformly at random from $\mathbf{Z} / a \mathbf{Z}$ or if $x_{a}^{*} \leftarrow x \bmod a$. We demonstrate that if $\mathcal{A}$ has significant probability in distinguishing both input types, then $C$ can use $\mathcal{A}$ to distinguish distributions $U$ and $\mathcal{V}$. We describe the steps of $C$ below:

$C(a, b, x)$ :

1. Set $x_{b} \leftarrow x \bmod b$

2. Sample $\beta^{*} \stackrel{\$}{\leftarrow}\{0,1\}$

3. If $\beta^{*}=0$, then $x_{a}^{*} \stackrel{\$}{\leftarrow} / a \mathbf{Z}$

4. Else if $\beta^{*}=1$, then $x_{a}^{*} \leftarrow x \bmod a$

5. $\beta^{\prime} \leftarrow \mathcal{A}\left(a, b, x_{b}, x_{a}^{*}\right)$

6. If $\beta=\beta^{\prime}$ return 1

7. Else return 0 .

If $C$ gets as input an element of $U$ whatever the value of $\beta^{*}, x_{a}^{*}$ follows the uniform distribution modulo $a$ and is independent of $x_{b}$. So $\mathcal{A}$ 's success probability in outputting $\beta^{\prime}$ equal to $\beta^{*}$ is $1 / 2$.

$$
\operatorname{Pr}\left[\mathcal{A}\left(a, b, x_{b}, x_{a}^{*}\right) \rightarrow \beta^{*} \mid(a, b, x) \hookleftarrow \mathcal{U}\right]=1 / 2
$$

and so

$$
\operatorname{Pr}[\mathcal{C}(u) \rightarrow 1]=1 / 2
$$

On the other hand if $(a, b, x) \hookleftarrow \mathcal{V}$, then $C$ outputs 1 if $\mathcal{A}$ guesses the correct bit $\beta^{*}$ (when its inputs are either in $u_{\mathcal{A}}$ or $\mathcal{V}_{\mathcal{A}}$ as expected).

$$
\operatorname{Pr}[C(\mathcal{V}) \rightarrow 1]=\operatorname{Pr}\left[\mathcal{A} \rightarrow \beta^{*} \mid(a, b, x) \hookleftarrow \mathcal{V}\right]
$$

And so

$$
\begin{aligned}
|\operatorname{Pr}[\Theta(\mathcal{U}) \rightarrow 1]-\operatorname{Pr}[\mathcal{C}(\mathcal{V}) \rightarrow 1]| & =\left|\operatorname{Pr}\left[\mathcal{A} \rightarrow \beta^{*} \mid(a, b, x) \hookleftarrow \mathcal{V}\right]-1 / 2\right| \\
& =1 / 2 \cdot\left|\operatorname{Pr}\left[\mathcal{A}\left(u_{\mathcal{A}}\right) \rightarrow 1\right]-\operatorname{Pr}\left[\mathcal{A}\left(\mathcal{V}_{A}\right) \rightarrow 1\right]\right| .
\end{aligned}
$$

Since distributions $u$ and $v$ are at statistical distance $\epsilon$, it holds that $\mid \operatorname{Pr}[\mathcal{C}(u) \rightarrow$ $1]-\operatorname{Pr}[\mathcal{C}(\mathcal{V}) \rightarrow 1] \mid \leq \epsilon$, and so for any algorithm $\mathcal{A}$ as above:

$$
\left|\operatorname{Pr}\left[\mathcal{A}\left(u_{\mathcal{A}}\right) \rightarrow 1\right]-\operatorname{Pr}\left[\mathcal{A}\left(\mathcal{V}_{A}\right) \rightarrow 1\right]\right| \leq 2 \epsilon .
$$

Thus the statistical distance between $U_{\mathcal{A}}$ and $\mathcal{V}_{\mathcal{A}}$ is smaller than $2 \epsilon$, which implies that even given $x \bmod b$, the value of $x \bmod a$ remains at negligible statistical distance $2 \epsilon$ of the uniform distribution modulo $a$, which concludes the proof. 


\section{Proof of Theorem 2}

In this section, we prove the following theorem.

Theorem 1. The protocol described in Figure 8 is a statistical zero-knowledge proof of knowledge with soundness $2^{-\ell}$, as long as $\ell$ is polynomial and $\ell S / A$ is negligible.

Proof. We prove completeness, soundness and zero-knowledge. Completeness follows easily by observing that when $\left(\left(\mathrm{pk},\left(c_{1}, c_{2}\right), Q_{1}\right) ;\left(x_{1}, r\right)\right) \in R_{\mathrm{CL}-\mathrm{DL}}$, for any $k \in\{0,1\}$ the values computed by an honest prover will indeed verify the four relations checked by the verifier. For soundness, the protocol is in fact special sound. Indeed notice that for committed values $t_{1}, t_{2}, t_{3}$, if a prover $P^{*}$ can answer correctly for two different values of $k$, he must be able to answer to challenges 0 and 1 with $u_{1}, u_{2}$ and $u_{1}^{\prime}, u_{2}^{\prime}$, where $u_{1}$ and $u_{1}^{\prime}$ are smaller than $A+S-1$, such that $u_{2} P=u_{2}^{\prime} P-Q$, $\mathrm{pk}^{u_{1}} f^{u_{2}} c_{2}=\mathrm{pk}^{u_{1}^{\prime}} f^{u_{2}^{\prime}}$ and $g_{q}^{u_{1}} c_{1}=g_{q}^{u_{1}^{\prime}}$. Let $\sigma_{1} \leftarrow u_{1}^{\prime}-u_{1}$, $\sigma_{2} \leftarrow u_{2}^{\prime}-u_{2} \bmod q$; we obtain $g_{q}^{\sigma_{1}}=c_{1}, \sigma_{2} P=Q$ and $\mathrm{pk}^{\sigma_{1}} f^{\sigma_{2}^{\prime}}=c_{2}$. Thus $P^{*}$ can easily compute $x_{1} \leftarrow \sigma_{2} \bmod q$ and $r \leftarrow \sigma_{1}$ in $\mathbf{Z}$.

While this gives a soundness error of $1 / 2$, the soundness is amplified to $2^{-\ell}$ by repeating the protocol sequentially $\ell$ times.

For zero-knowledge, we must exhibit a simulator $\delta$ which, given the code of some verifier $V^{*}$, produces a transcript indistinguishable from that which would be produced between $V^{*}$ and an honest prover $P$ (proving the knowledge of a tuple in $R_{\mathrm{CL}-\mathrm{DL}}$ ) without knowing the witnesses $\left(x_{1}, r\right)$ for (pk, $\left.\left(c_{1}, c_{2}\right), Q_{1}\right)$ in the relation $R_{\mathrm{CL}-\mathrm{DL}}$.

The potentially malicious verifier may use an adaptive strategy to bias the choice of the challenges to learn information about $\left(r, x_{1}\right)$. This implies that challenges may not be randomly chosen, which must be taken into account in the security proof.

We describe an expected polynomial time simulation of the communication between a prover $P$ and a malicious verifier $V^{*}$ for one round of the proof. Since the simulated round may not be the first round, we assume $V^{*}$ has already obtained data, denoted by hist, from previous interactions with $P$. Then $P$ sends the commitments $t_{1}, t_{2}, t_{3}$ and $V^{*}$ chooses possibly using hist and $t_{1}, t_{2}, t_{3}$ - the challenge $k\left(t_{1}, t_{2}, t_{3}\right.$, hist).

Description of the simulator: Consider the simulator $\mathcal{S}$ which simulates a given round of identification as follows:

1. $\mathcal{S}$ chooses random values $\bar{k} \in\{0,1\}, \bar{u}_{1} \in[S-1, A-1]$ and $\bar{u}_{2} \in \mathbf{Z} / q \mathbf{Z}$.

2. $\mathcal{S}$ computes $\overline{t_{1}} \leftarrow \mathrm{pk}^{\bar{u}_{1}} f^{\bar{u}_{2} / c_{2}^{\bar{k}}} ; \overline{t_{2}} \leftarrow\left[\bar{u}_{2}\right] P-[\bar{k}] Q$ and $\overline{t_{3}} \leftarrow g_{q}^{\bar{u}_{1}} / c_{1}^{\bar{k}}$, and sends $\overline{t_{1}}, \overline{t_{2}}$ and $\overline{t_{3}}$ to $V^{*}$.

3. $\&$ receives $k\left(\overline{t_{1}}, \overline{t_{2}}, \overline{t_{3}}\right.$, hist $)$ from $V^{*}$.

4. If $k\left(\overline{t_{1}}, \overline{t_{2}}, \overline{t_{3}}\right.$, hist $) \neq \bar{k}$ then return to step 1 , else return $\left(\overline{t_{1}}, \overline{t_{2}}, \overline{t_{3}}, \bar{k}, \bar{u}_{1}, \bar{u}_{2}\right)$.

To demonstrate that the proof is indeed zero-knowledge, we need to justify that the distribution output by the simulator is statistically close to that output in a real execution of the protocol, and that the simulation runs in expected polynomial time.

Intuitively, sampling the randomness $r$ from a large enough distribution - i.e. as long as $S<<A$ - ensures that the distribution of $t_{1}, t_{2}, t_{3}$ in a real execution is statistically close $\mathrm{e}^{1}$ to that in a simulated execution.

\footnotetext{
1 The distributions cannot be distinguished by any algorithm, even using an infinite computational power, but only accessing a polynomial number of triplets of both distributions
} 
The analysis of the above statistical distance $\Sigma$ between the distribution of tuples output by the simulator and that of tuples output by a real execution of the protocol is quite tedeous and similar to that of GPS06. We do not provide the details here but applying their analysis to our setting allows us obtain the following bound:

$$
\Sigma<\frac{8 S}{A}
$$

Thus the real and simulated distributions are statistically indistinguishable if $S / A$ is negligible.

Running time of the Simulator: We now need to ensure that the simulator runs in expected polynomial time. To see this, notice that step 3 outputs a tuple $\left(\overline{t_{1}}, \overline{t_{2}}, \overline{t_{3}}, \bar{k}, \bar{u}_{1}, \bar{u}_{2}\right)$ if $k\left(\overline{t_{1}}, \overline{t_{2}}, \overline{t_{3}}\right.$, hist $)=\bar{k}$. Since $\bar{k}$ is sampled at random from $\{0,1\}$, the expected number of iterations of the loop is 2 . Therefore the complexity of the simulation of all $\ell$ rounds is $O(\ell)$.

Thus if $\ell S / A$ is negligible and $\ell$ is polynomial, the protocol is statistically zero-knowledge.

\section{Lindell's new interactive assumption}

In order to prove the security of his 2-party ECDSA, Lindell introduced in [Lin17 the following ad hoc interactive assumption, called Paillier-EC assumption. It is defined via the following random experiment.

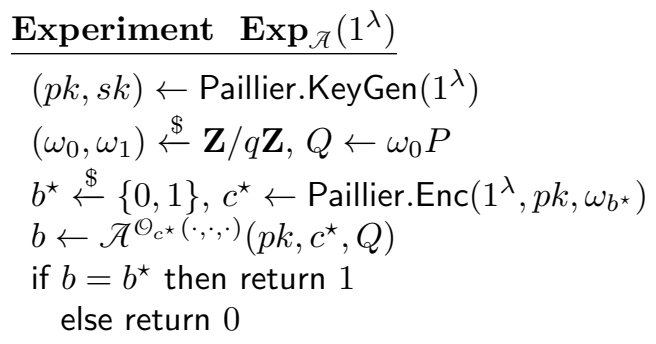

where $1 \leftarrow \mathcal{\Theta}_{c^{\star}}\left(c^{\prime}, \alpha, \beta\right)$ if and only if Paillier.Dec $\left(1^{\lambda}, s k, c^{\prime}\right)=\alpha+\beta \omega_{b^{\star}} \bmod q$ and $\mathcal{G}$ stops after the first time it returns 0 .

The Paillier-EC assumption is hard if for every probabilistic polynomial-time adversary $\mathcal{A}$ there exists a negligible function $\nu$ such that $\operatorname{Pr}\left[\operatorname{Exp}_{\mathcal{A}}\left(1^{\lambda}\right)=1\right] \leq \frac{1}{2}+\nu(n)$. 


\section{Two-Party ECDSA from HSM}

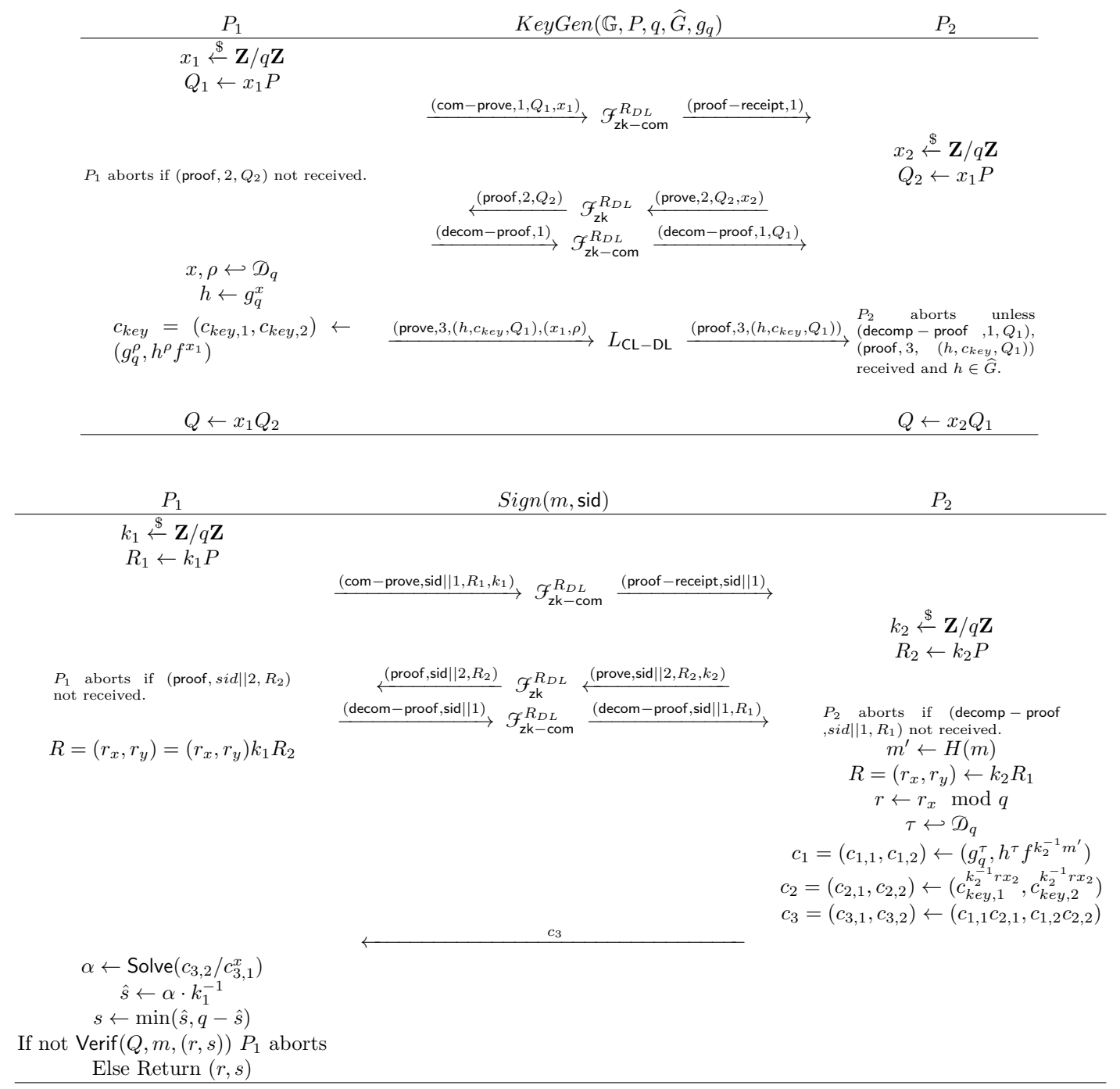

